\title{
New data on the spider fauna (Aranei) of Dagestan, Russia
}

\section{Новые данные по фауне пауков (Aranei) Аагестана, Россия}

\author{
Alexander V. Ponomarev ${ }^{1 *}$, Magomed A. Aliev², \\ Gadzhimurad N. Khabiev ${ }^{3}$, Vladimir Yu. Shmatko ${ }^{1}$ \\ Александр В. Пономарёв ${ }^{1}$, Магомед А. Алиев ${ }^{2}$, \\ ГаАжимураА Н. Хабиев ${ }^{3}$, ВАадимир Ю. Шматко ${ }^{1}$
}

\footnotetext{
${ }^{1}$ Southern Scientific Centre RAS, Chekhov str., 41, Rostov-on-Don, 344006, Russia.

2 Dagestan State University, Batyraya str., 4a, 2nd floor, Makhachkala, 367000, Russia.

Caspian Institute of Biological Resources, Dagestan Scientific Center of RAS, M. Gadzhieva str., 45, Makhachkala, 367000, Russia.

${ }^{1}$ Южный научный центр РАН, пр. Чехова, 41, Ростов-на-Дону 344006, Россия.

2 Дагестанский государственный университет, ул. Батырая, 4a, 2 этаж, Махачкала, 367000, Россия.

${ }^{3}$ Прикаспийский институт биоресурсов ДНЦ РАН, ул. М. Гаджиева, 45, Махачкала, 367000, Россия.

* Correspinding author; e-mail: ponomarev1952@mail.ru
}

KEY WORDS: Araneae, faunistics, new findings, northern Caucasus.

КЛЮЧЕВЫЕ СЛОВА: Araneae, фаунистика, новые находки, северный Кавказ.

ABSTRACT. An annotated list of 223 spider species in 28 families recorded from the territory of the Republic of Dagestan in 2009-2018 is given. Fourteen species (Dictyna armata, D. ottoi, Berlandina saraevi, Nomisia conigera, Nomisia ripariensis, Talanites dunini, Pholcus sogdianae, Aelurillus concolor, Chalcoscirtus tanasevichi, Chinattus caucasicus, Phlegra lineata, Yllenus zyuzini, Xysticus kaznakovi, X. loeffleri) are reported from the territory of Russia for the first time; 31 species are new for the fauna of Dagestan. Illustrations are provided for 13 species that are new to the fauna of Russia, five species known from the territory of Russia from one/two localities and one that is yet undeteremined.

How to cite this article: Ponomarev A.V., Aliev M.A., Khabiev G.N., Shmatko V.Yu. 2019. New data on the spider fauna (Aranei) of Dagestan, Russia // Arthropoda Selecta. Vol.28. No.2. P.309-334. doi: 10.15298/arthsel. 28.2.14

РЕЗЮМЕ. Приводится аннотированный список 223 видов пауков из 28 семейств отмеченных с территории Республики Дагестан в 2009-2018 годах. Четырнадцать видов (Dictyna armata, D. ottoi, Berlandina saraevi, Nomisia conigera, Nomisia ripariensis, Talanites dunini, Pholcus sogdianae, Aelurillus concolor, Chalcoscirtus tanasevichi, Chinattus caucasicus, Phlegra lineata, Yllenus zyuzini, Xysticus kaznakovi, X. loeffleri) впервые отмечаются на территории России; 31 видов являются новыми для фауны Дагестана. Проиллюстрированы 13 видов новых для фауны России, 5 видов известных с территории России из одного или двух локалитетов и один окончательно неопределенный вид.

\section{Introduction}

Special research on the araneofauna of Dagestan started in 2007 [Ponomarev, Khalidov, 2007; Ponomarev et al., 2008, 2011a,b; Ponomarev, Alieva, 2008, 2010; Abdurakhmanov, Alieva, 2009, 2011], and the results were summarized in the book by Abdurakhmanov et al. [2012]. A total of 397 species in 163 genera and 31 families was listed in that book. However, as was stressed upon by the authors of the latter book, their data were not complete, especially as far as the poorly studied Linyphiidae and Salticidae concern. Later, 17 species and the family Segestriidae from the Caspian coast and Sarykum sand dunes were added to the species list [Ponomarev, Dvadnenko, 2013; Ponomarev, Abdurakhmanov, 2014; Ponomarev et al., 2017b]. To date, a list of the Dagestan spiders consists of 414 species in 32 families.

The present paper presents additional faunistic records to and new distributional data on the spider fauna of Dagestan.

\section{Material and methods}

The present study is based on the spider material collected by M.A. Aliev (M.A.), G.H. Khabiev (G.Kh.), E.V. Il'ina (E.I.) and students of the biological faculty of the Dagestan State University from the territory of the Republic of Dagestan (Russia) from 17 districts and Makhachkala City and its vicinity in 2009-2018. All the collecting localities and their coordinates are described below:

Akusha District:

1 - Usisha $\left(42.251378^{\circ} \mathrm{N}, 47.389419^{\circ} \mathrm{E}\right)$.

Babayurt District:

2 - Babayurt $\left(43.603249^{\circ} \mathrm{N}, 46.774474^{\circ} \mathrm{E}\right)$; 
3 - Terek River delta, "Terskaya" biological station $\left(43.602028^{\circ} \mathrm{N}, 47.508408^{\circ} \mathrm{E}\right)$.

Buynaksk District:

4 - Chirkey $\left(42.990153^{\circ} \mathrm{N}, 46.914414^{\circ} \mathrm{E}\right)$;

$5-3 \mathrm{~km} \mathrm{NW}$ of Erpeli $\left(42.798304^{\circ} \mathrm{N}, 47.004134^{\circ} \mathrm{E}\right)$.

Gumbetovsky District:

6 - Chirkata $\left(42.806063^{\circ} \mathrm{N}, 46.718503^{\circ} \mathrm{E}\right)$;

7 - Ingishi $\left(42.776324^{\circ} \mathrm{N}, 46.559250^{\circ} \mathrm{E}\right)$.

Derbent District:

8 - nr. Gelinbatan $\left(41.922582^{\circ} \mathrm{N}, 48.222867^{\circ} \mathrm{E}\right)$.

Kazbekovsky District:

9 - Dylym $\left(43.063704^{\circ} \mathrm{N}, 46.632083^{\circ} \mathrm{E}\right)$;

10 - Dubki $\left(42.991010^{\circ} \mathrm{N}, 46.865732^{\circ} \mathrm{E}\right)$;

11 - between Gertma and Akhsu $\left(42.92100008^{\circ} \mathrm{N}\right.$, $\left.46.68950991^{\circ} \mathrm{E}\right)$

12 - Salatau Ridge, above Gertma $\left(42.92119626^{\circ} \mathrm{N}\right.$, $\left.46.68960231^{\circ} \mathrm{E}\right)$

Kaytagsky District:

13 - Karatsan $\left(42.086487^{\circ} \mathrm{N}, 47.875730^{\circ} \mathrm{E}\right)$.

Karabudakhkent District:

$14-15 \mathrm{~km} \mathrm{~W}$ of Gubden $\left(42.53629692^{\circ} \mathrm{N}, 47\right.$. $\left.39692388^{\circ} \mathrm{E}\right)$;

15 - Gurbuki $\left(42.609722^{\circ} \mathrm{N}, 47.602778^{\circ} \mathrm{E}\right)$.

Khunzakh District:

16 - Khunzakh $\left(42.626701 \mathrm{~N}^{\circ}, 47.595823^{\circ} \mathrm{E}\right)$.

Kisilyurt District:

17 - Shushanovka $\left(43.242987^{\circ} \mathrm{N}, 46.976579^{\circ} \mathrm{E}\right)$.

Kumtorkalinsky District:

18 - Almalo $\left(43.138773^{\circ} \mathrm{N}, 47.211468^{\circ} \mathrm{E}\right)$;

19 - Sarykum sand dune $\left(43.004652^{\circ} \mathrm{N}, 47.238217^{\circ} \mathrm{E}\right)$;

20 - Karaman $\left(43.049602^{\circ} \mathrm{N}, 47.441298^{\circ} \mathrm{E}\right)$;

21 - nr. Kumtorkala railway station, Shura-Ozen River valley $\left(43.000523^{\circ} \mathrm{N}, 47.239608^{\circ} \mathrm{E}\right)$;

22 - Narat Tyube Ridge $\left(43.002609^{\circ} \mathrm{N}, 47.202866^{\circ} \mathrm{E}\right)$.

Magaramkent District:

23 - Samur forest $\left(41.853258^{\circ} \mathrm{N}, 48.556194^{\circ} \mathrm{E}\right)$;

24 - Samur River delta $\left(41.866772^{\circ} \mathrm{N}, 48.556253^{\circ} \mathrm{E}\right)$,

25 - Chakh-Kazmalyar $\left(41.786668^{\circ} \mathrm{N}, 48.490247^{\circ} \mathrm{E}\right)$.

Nogaysky District:

26 - Leninaul $\left(44.205227^{\circ} \mathrm{N}, 46.030404^{\circ} \mathrm{E}\right)$;

27 - Terekli-Mekteb $\left(44.160017^{\circ} \mathrm{N}, 45.809769^{\circ} \mathrm{E}\right)$;

$28-20 \mathrm{~km} \mathrm{~S}$ of Chervlennye Buruny $\left(44.019625^{\circ} \mathrm{N}\right.$, $\left.45.626715^{\circ} \mathrm{E}\right)$

Rutul District:

29 - Kufa $\left(41.567194^{\circ} \mathrm{N}, 47.360092^{\circ} \mathrm{E}\right)$.

Suleyman-Stalsky District:

30 - Novaya Maka $\left(41.790573^{\circ} \mathrm{N}, 48.353630^{\circ} \mathrm{E}\right)$.

Tabasaransky District:

31 - Sirtich $\left(41.808106^{\circ} \mathrm{N}, 48.180833^{\circ} \mathrm{E}\right)$.

Shamilsky District:

32 - Tidib $\left(42.393845^{\circ} \mathrm{N}, 46.546769^{\circ} \mathrm{E}\right)$.

Vicinity of Makhachkala:

33 - Makhachkala $\left(42.965619^{\circ} \mathrm{N}, 47.520647^{\circ} \mathrm{E}\right)$.

34 - Leninkent $\left(42.968302^{\circ} \mathrm{N}, 47.335658^{\circ} \mathrm{E}\right)$;

35 - Tarki $\left(42.946194^{\circ} \mathrm{N}, 47.495555^{\circ} \mathrm{E}\right)$;

36 - Tarki-Tau Mt. $\left(42.946954^{\circ} \mathrm{N}, 47.489281^{\circ} \mathrm{E}\right)$;

37 - Turali $\left(42.948042^{\circ} \mathrm{N}, 47.578690^{\circ} \mathrm{E}\right)$;

$38-18 \mathrm{~km}$ W of Makhachkala, Talgi Canyon (42. $\left.876402^{\circ} \mathrm{N}, 47.441217^{\circ} \mathrm{E}\right)$.

Different collecting methods were used: viz., hand collecting (with the aid of forceps, aspirator or sweeping net), pitfall traps and siefters. In total, some 1600 spider specimens were collected, of which more than 1000 were adults. The material is currently stored in the personal collection by A.V. Ponomarev but soon will be transferred to the collec- tion of the Zoological Museum of the Moscow State University (Moscow, Russia).

Digital images were made in the Southern Scientific Centre of the Russian Academy of Science (Rostov-on-Don) by measn of a Mikmed-6 microscope with a digital camera SONYNEXC3 16.2mp and microphotonozzle MFN-12.

\section{List of species}

\section{AGELENIDAE}

Agelena labyrinthica (Clerck, 1758)

RECORDS. Russia, DAGESTAN: Buynaksk [Ponomarev et al., 2008]; Kurush, Kondik, Chuvek, Tsuduk [Abdurakhmanov, Alieva, 2009, 2011]; Sergokala, Tselyagyun [Abdurakhmanov, Alieva, 2011]; Ingishi [Abdurakhmanov et al., 2012]; Sarykum sand dune [Ponomarev, Abdurakhmanov, 2014].

MATERIAL. Russia, Dagestan: $1 \sigma^{7}$, [13], forest, 17.07.2013 (Galimova); 1 OT$^{7}$, [29], 20.07.2017, (M.A.).

Eratigena agrestis (Walckenaer, 1802)

RECORDS. Russia, Dagestan: Verkhny Karanay [Ponomarev, Khalidov, 2007: Tegenaria]; Nizhnee Kazanishche [Ponomarev et al., 2008: Tegenaria]. mova).

MATERIAL. Russia, Dagestan: 3 우, [33], 1.12 .2012 (Gali-

Pireneitega ovtchinnikovi Kovblyuk, Kastrygina, Marusik et Ponomarev, 2013

MATERIAL. Russia, Dagestan: 1 + , [9], 3.08 .2012 (G.Kh.).

COMMENTS. The new record for the fauna of Dagestan. It is widespread in the northern and western Caucasus [Kovblyuk et al., 2013].

Tegenaria lyncea Brignoli, 1978

RECORDS. Russia, Dagestan: Verkhnee Kazanishche, Verkhny Karanay [Ponomarev, Khalidov, 2007: Malthonica sp.; Khalidov, Ponomarev, 2008: Malthonica sp.]; Aglobi [Abdurakhmanov, Alieva, 2009: Malthonica sp.; 2011: Malthonica lyncea]; Gasha, Makhachkala, Tselyagyun [Ponomarev, Alieva, 2010: Malthonica lyncea; Abdurakhmanov, Alieva, 2011: Malthonica lyncea]; Nizhnee Kazanishche [Ponomarev et al., 2011b: Malthonica lyncea].

MATERIAL. Russia, Dagestan: 1 \%, [9], 20.07 .2012 (G.Kh.); 2 O $^{7} \sigma^{7},[19], 06.2013$ (G.Kh.).

\section{AMAUROBIIDAE}

Amaurobius erberi (Keyserling, 1863)

Figs 1-2.

Ciniflo erberi Keyserling, 1863: 373, pl. 10, figs 5-6 ( $\left.\left.\mathrm{O}^{7}+\right)\right)$

Amaurobius erberi: L. Koch, 1868: 21, figs 9-10 (О7\%)

Amaurobius erberi: Thaler, Knoflach, 1995: 57, figs 2, 17, 20, $27,44-45\left(\mathrm{O}^{7}+\right)^{2}$

MATERIAL. Russia, Dagestan: 1 , [33], Leninsky Komsomol Park, 1.01.2012 (G.Kh.).

COMMENTS. The new record for the fauna of Dagestan. In the territory of Russia, it has been reported from the Crimea [Mikhailov, 2013]. The specimens of this species collected from Russia have never been illustrated. Thus, in order to prove that our record is correct, the epigyne of the female studied is illustrated (Figs 1-2). 

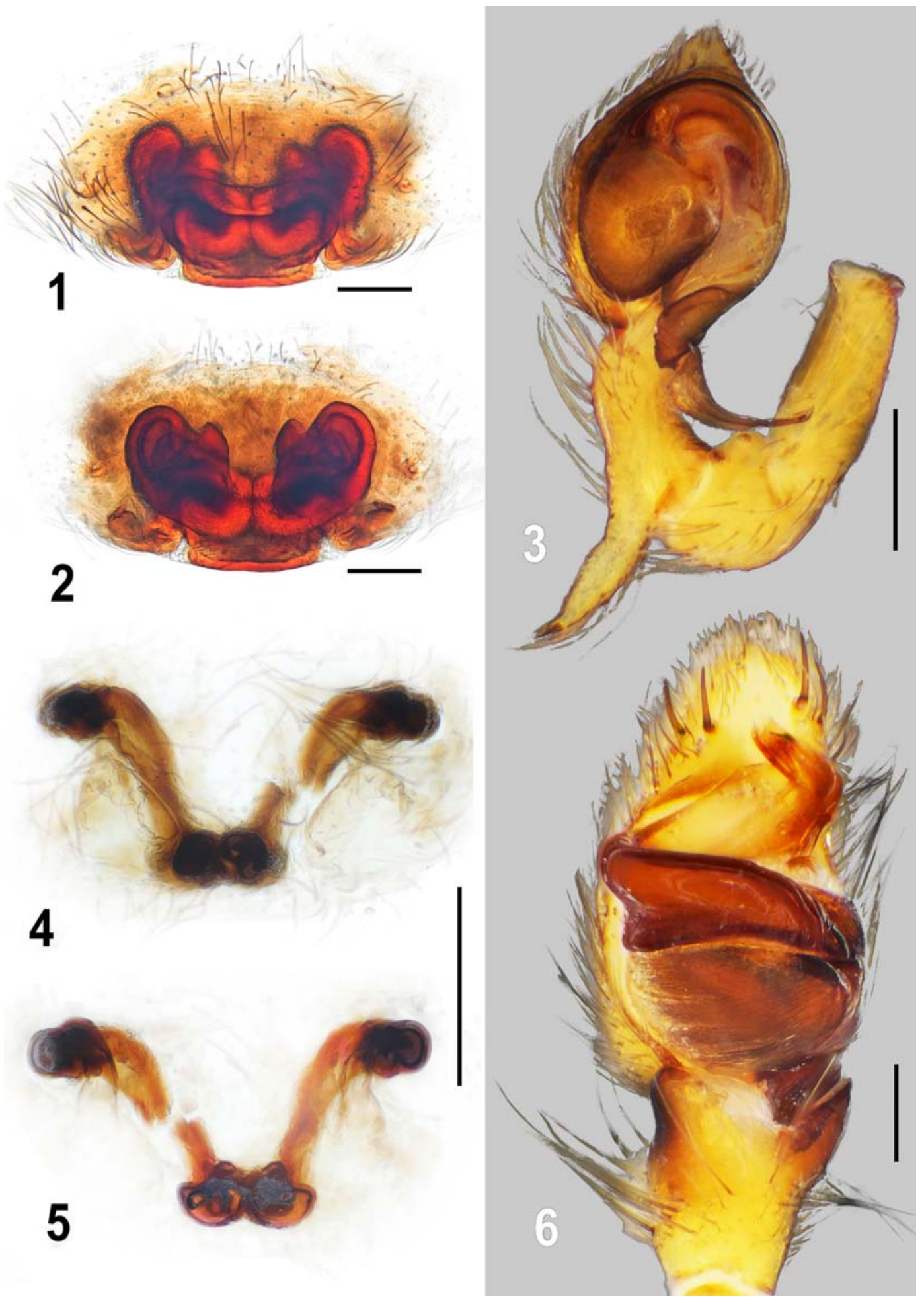

Figs 1-6. Copulatory organs of Amaurobius erberi (Keyserling, 1863) (1, 2), Dictyna armata Thorell, 1875 (3), Dictyna ottoi Marusik et Koponen, 2017 (4, 5), Berlandina saraevi Ponomarev, 2008 (6): 1, 4 - epigyne, ventral view; 2, 5 — spermathecae, dorsal view; 3, 6 male palp, ventral view. Scale bar: $0.25 \mathrm{~mm}$.

Рис. 1-6. Копулятивные органы Amaurobius erberi (Keyserling, 1863) (1, 2), Dictyna armata Thorell, 1875 (3), Dictyna ottoi Marusik et Koponen, 2017 (4, 5), Berlandina saraevi Ponomarev, 2008 (6): 1, 4 — эпигина, вид снизу; 2, 5 - сперматека, вид сверху; 3,6 - пальпа самца, вид снизу. Масштаб: 0,25 мм. 


\section{ANYPHAENIDAE}

Anyphaena accentuata (Walckenaer, 1802)

RECORDS. Russia, DAgESTAN: Samur forest, Irganay [Abdurakhmanov, Alieva, 2009, 2011].

MATERIAL. Russia, Dagestan: 1 + , [9], 6.07 .2011 (G.Kh.); 1 o', [9], 3.08.2012 (G.Kh.); 1 O', [23], 1.04.2017 (M.A.).

\section{ARANEIDAE}

Agalenatea redii (Scopoli, 1763)

RECORDS. Russia, DAGESTAN: Dagestan [Kroneberg, 1875: Epeira sollers]; Makhachkala, Maydanskoe [Abdurakhmanov, Alieva, 2009, 2011]; Sarykum sand dune [Ponomarev et al., 2011b, 2017b; Ponomarev, Abdurakhmanov, 2014].

MATERIAL. Russia, Dagestan: 1 +, [13], forest, 28.06.2013 (Galimova); 2 우, [19], 04-05.2014 (G.Kh.); 2 우, [31], 30.04.2017 (M.A.); 1 \%, [8], 28.05.2017 (M.A.)

\section{Araneus angulatus Clerck, 1758}

RECORDS. Russia, Dagestan: Dzhaba, Dubki [Ponomarev et al., 2008]; Makhachkala, Khvarshi [Abdurakhmanov, Alieva, 2009, 2011]; Karabudakhkent, Novyi Kumukh, Nizhnee Kazanishche, Chapaevo [Ponomarev et al., 2011b];

MATERIAL. Russia, Dagestan: 1 ㅇ, [3], 06.2011 (G.Kh.); 1 +, [13], 24.07.2012 (Galimova); 1 +, [5], $730 \mathrm{~m}$ a.s.1., 19.06.2017 (M.A.).

\section{Araneus diadematus Clerck, 1758}

RECORDS. Russia, Dagestan: Verkhnee Kazanishche [Ponomarev, Khalidov, 2007; Khalidov, Ponomarev, 2008]; Aimaki [Ponomarev et al., 2008b]; Makhachkala, Nizhnee Kazanishche [Ponomarev et al., 2008, 2011b]; Makhachkala, Inkhokvari, Khonokh, Khvarshi [Abdurakhmanov, Alieva, 2009, 2011]; Tselyagyun [Abdurakhmanov, Alieva, 2011]; Sulak, Khasavyurt [Ponomarev et al., 2011b]; Agrakhan Peninsula [Ponomarev, Abdurakhmanov, 2014].

MATERIAL. Russia, Dagestan: 10 +9, [9], 4.09.2013 (G.Kh.).

Araniella cucurbitina (Clerck, 1758)

RECORDS. Russia, DAGESTAN: Verkhny Karanay [Ponomarev, Khalidov, 2007]; Erpeli, Verkhnee Kazanishche [Ponomarev, Khalidov, 2007; Khalidov, Ponomarev, 2008; Ponomarev et al., 2008]; Verkhny Gunib, Khuchni [Ponomarev et al., 2008b]; Kondik, Chuvek, Khvarshi [Abdurakhmanov, Alieva, 2009, 2011]; Nizhnee Kazanishche [Ponomarev et al., 2011b]; Mamedkala [Abdurakhmanov et al., 2012]; Sarykum sand dune [Ponomarev et al., 2017b].

MATERIAL. Russia, Dagestan: 1 , [9], 6.07.2011 (G.Kh.); 1 $\mathrm{o}^{7}$, [35], 6.06.2013 (G.Kh.); 1 + , [5], meadow, $730 \mathrm{~m}$ a.s.1., 19.06.2017 (M.A.); $1 \mathrm{O}^{7}$, [11], 16.07.2017 (M.A.).

Araniella opisthographa (Kulczyński, 1905)

RECORDS. Russia, Dagestan: Samur forest [Abdurakhmanov, Alieva, 2009, 2011]; Gasha, Turali, Tselyagyun [Abdurakhmanov, Alieva, 2011]

MATERIAL. Russia, Dagestan: $1 \sigma^{7}, 1 \%,[10], 8.07 .2013$ (A.A. Omarov, I.M. Ushanov).

Argiope bruennichi (Scopoli, 1772)

RECORDS. Russia, Dagestan: Nizhny Dzhengutai [Ponomarev et al., 2008]; Makhachkala [Abdurakhmanov, Alieva, 2009, 2011]; Nizhnee Kazanishche, Chirkey, Novokuli, Novyi Kumukh, Talgi Canyon [Ponomarev et al., 2011b]; Aglobi [Abdurakhmanov, Alieva, 2011].
MATERIAL. Russia, DAGESTAN: $1 \sigma^{7}, 4$ 우 [13], 24.07.2012 (Galimova); 1 ․, [9], 6.08.2012 (G.Kh.).

Argiope lobata (Pallas, 1772)

RECORDS. Russia, Dagestan: Aglobi [Abdurakhmanov, Alieva, 2009, 2011: in both cases Argiope sp.]; Khidib [Ponomarev, Alieva, 2010]; Bolshie Turali, Izberbash [Ponomarev, Alieva, 2010; Abdurakhmanov, Alieva, 2011]; Tyuleniy Island [Ponomarev et al., 2011a]; Chirkey, Nizhnee Kazanishche, Chapaevo [Ponomarev et al., 2011b]; Sarykum sand dune [Ponomarev et al., 2017b].

MATERIAL. Russia, Dagestan: 1 q juv., [2], 23.06.2011 (G.Kh.); 1 + , [3], 24.06 .2012 (G.Kh.).

Cyclosa conica (Pallas, 1772)

RECORDS. Russia, Dagestan: Verkhnee Kazanishche, Verkhny Karanay [Ponomarev, Khalidov, 2007; Khalidov, Ponomarev, 2008; Ponomarev et al., 2008]. (G.Kh.)

MATERIAL. Russia, Dagestan: 1 q, [23], 16-18.06.2014

Gibbaranea bituberculata (Walckenaer, 1802)

RECORDS. Russia, Dagestan: Buynaksk Distr. [Ponomarev et al., 2008]; Bryanskaya Kosa [Ponomarev et al., 2011a]; Sarykum sand dune [Ponomarev et al., 2011b, 2017b; Ponomarev, Abdurakhmanov, 2014].

MATERIAL. Russia, Dagestan: 1 \%, [13], 28.04 .2012 (Galimova).

Gibbaranea ullrichi (Hahn, 1835)

RECORDS. Russia, Dagestan: Yuzhno-Sukhokumsk [Abdurakhmanov et al., 2012].

MATERIAL. Russia, Dagestan: 1 + , [18], 4.06 .2017 (M.A.).

Hypsosinga albovittata (Westring, 1851)

RECORDS. Russia, Dagestan: Yuzhno-Sukhokumsk [Abdurakhmanov et al., 2012].

MATERIAL. Russia, Dagestan: 1 ㅇ, [25], 28.05 .2017 (M.A.); 1 ․ [8], 28.05.2017 (M.A.); 2 우, [27], 12.06.2017 (M.A.).

Hypsosinga pygmaea (Sundevall, 1831)

RECORDS. Russia, Dagestan: Tyuleniy Island [Abdurakhmanov et al., 2012].

MATERIAL. Russia, Dagestan: 1 + , [26], 11.06.2017, (M.A.); 2 우, [27], 12.06.2017 (M.A.).

\section{Larinioides folium (Schrank, 1803)}

RECORDS. Russia, Dagestan: Nordovyi Island, Tyuleniy Island [Ponomarev et al., 2011a]; Kochubei, Makhachkala [Ponomarev et al., 2011b], Inchkhe [Abdurakhmanov et al., 2012].

MATERIAL. Russia, Dagestan: 1 \%, [9], 27.07.2012 (G.Kh.); 1 ․, [19], 14.05.2014 (G.Kh.).

\section{Mangora acalypha (Walckenaer, 1802)}

RECORDS. Russia, Dagestan: Erpeli, Verkhnee Kazanishche, Verkhny Karanay [Ponomarev, Khalidov, 2007; Khalidov, Ponomarev, 2008; Ponomarev et al., 2008]; Nizhnee Kazanishche [Ponomarev et al., 2011b]; Samur forest, Maydanskoe, Kondik [Abdurakhmanov, Alieva, 2009, 2011]; Turali, Gasha, Sergokala [Abdurakhmanov, Alieva, 2011]; Bryanskaya Kosa [Ponomarev et al., 2011a]; Yuzhno-Sukhokumsk [Abdurakhmanov et al., 2012]; Sarykum sand dune [Ponomarev, Abdurakhmanov, 2014; Ponomarev et al., 2017b].

MATERIAL. Russia, Dagestan: 2 우, [19], 14.05 .2014 (G.Kh.); 1 , [25], 28.05.2017 (M.A.); 2 ㅇ, [23], 25.06.2017 (M.A.). 
Neoscona adianta (Walckenaer, 1802)

RECORDS. Russia, Dagestan: Nizhny Dzhengutai [Ponomarev et al., 2008]; Aglobi, Inkhokvari, Maydanskoe, Kondik, Chuvek, Khvarshi, Khonokh, Tsuduk [Abdurakhmanov, Alieva, 2009, 2011]; Gasha, Tselyagyun [Abdurakhmanov, Alieva, 2011]; Bryanskaya Kosa, Tyuleniy Island [Ponomarev et al., 2011a]; Erpeli, Nizhnee Kazanishche [Ponomarev et al., 2011b]; Yuzhno-Sukhokumsk [Abdurakhmanov et al., 2012].

MATERIAL. Russia, Dagestan: 1 + [19], 06.2009 (S. Gamzatova); 1 O$^{7}, 1$ ㅇ, [2], 14.06.2013 (G.Kh.); 1 O $^{7}, 2$ 우, [3], 06.2013 (Abdullaeva, Magomedova); $3 O^{\Upsilon} \sigma^{7}, 2$ 우, [27], 12.06.2017 (M.A.); $1 \sigma^{7}, 2$ + + , [29], 20.07.2017 (M.A.).

Nuctenea umbratica (Clerck, 1758)

RECORDS. Russia, Dagestan: Nizhny Dzhengutai, Verkhny Dzhengutai [Ponomarev et al., 2008]; Kondik [Abdurakhmanov, Alieva, 2009, 2011]; Buynaksk, Verkhny Gunib [Ponomarev et al., 2011b].

MATERIAL. Russia, Dagestan: 1 +, [9], 4.09 .2013 (G.Kh.).

Singa hamata (Clerck, 1758)

RECORDS. Russia, Dagestan: Tyuleniy Island [Ponomarev et al., 2011a].

MATERIAL. Russia, Dagestan: $1 \sigma^{7}, 4$ +o , [23], $16-$ 18.06.2014 (G.Kh.)

Singa semiatra L. Koch, 1867

RECORDS. Russia, Dagestan: Sarykum sand dune [Ponomarev et al., 2017b]

MATERIAL. Russia, Dagestan: 1 , [5], meadow, $730 \mathrm{~m}$ a.s.1., 19.06.2017 (M.A.); $1 \sigma^{7}$, [19], tidal saltmarsh, 28.04.2018 (M.A.).

COMMENTS. This species is rare in Dagestan and known from the following Russian regions: the Crimea, Taman Peninsula, vicinity of Gelendzhik (Krasnodar Region) [Ponomarev et al., 2017c].

Zilla diodia (Walckenaer, 1802)

RECORDS. Russia, DageSTAN: Verkhny Karanay [Ponomarev, Khalidov, 2007; Khalidov, Ponomarev, 2008]; Verkhny Dzhengutai [Ponomarev et al., 2008]; Gasha, Madzhalis [Abdurakhmanov, Alieva, 2011]; Nizhnee Kazanishche [Ponomarev et al., 2011b].

MATERIAL. Russia, Dagestan: 1 + , [9], 6.07 .2011 (G.Kh.); 1 P, [19], 04-05.2014 (G.Kh.).

\section{CHEIRACANTIIDAE}

Cheiracanthium gratum Kulczyński in Chyzer et Kulczyński, 1897

MATERIAL. Russia, Dagestan: $1 \sigma^{7}$, [21], sagebrush, 5.05 . 2013 (E.I.).

COMMENTS. The new record for the fauna of Dagestan. In Russia, it is known from Kalmykia [Tsvetkov et al., 2006], Volgograd Area [Ponomarev, Khnykin, 2013] and Stavropol Territory [Ponomarev et al., 2017a]; elsewhere the species is rare.

Cheiracanthium montanum L. Koch, 1878

RECORDS. Russia, Dagestan: Verkhnee Kazanishche [Ponomarev et al., 2008]; Tselyagyun [Abdurakhmanov, Alieva, 2011]; Bryanskaya Kosa, Tyuleniy Island [Ponomarev et al., 2011a]; Nizhnee Kazanishche [Ponomarev et al., 2011b]; Chechen Island [Ponomarev, Abdurakhmanov, 2014]; Sarykum sand dune [Ponomarev et al., 2017b].
MATERIAL. Russia, DagestAn: $10^{7},[21]$, gully slope with sagebrush, 12.05.2013 (E.I.); 2 O$^{\top} \sigma^{\top}, 1$ +, [19], 04-05.2014 (G.Kh.); 1 + , [27], 12.06.2017 (M.A.).

Cheiracanthium pennyi O. Pickard-Cambridge, 1873 RECORDS. Russia, Dagestan: Bryanskaya Kosa [Ponomarev et al., 2011a].

MATERIAL. RusSia, Dagestan: $1 \mathrm{O}^{\top 1}$, [2], 14.06.2013 (G.Kh.); $1 \mathrm{O}^{7}$, [8], 28.05.2017 (M.A.).

Cheiracanthium punctorium (Villers, 1789)

RECORDS. Russia, Dagestan: Buynaksk, Dzhaba [Ponomarev et al., 2008]; Tselyagyun [Abdurakhmanov, Alieva, 2011]; Nizhnee Kazanishche [Ponomarev et al., 2011b].

MATERIAL. Russia, Dagestan: $1 O^{7}$, [9], 3.08 .2012 (G.Kh.).

\section{DICTYNIDAE}

Brigittea latens (Fabricius, 1775)

MATERIAL. Russia, Dagestan: $2 O^{7} \sigma^{7}$, [30], nr. a pond, 28. 05.2017 (M.A.).

COMMENTS. The new record for the fauna of Dagestan. This species is widespread in Europe [Nentwig et al., 2018]; in the Caucasus, it is known from Azerbaijan [Mikhailov, 2013] and South Ossetia [Ponomarev, Komarov, 2015].

\section{Dictyna armata Thorell, 1875}

Fig. 3.

Dictyna armata Thorell, 1875: $72\left(\sigma^{7}\right)$

Dictyna armata: Marusik, Koponen, 2017: 45, figs 3a-d ( $\left.\sigma^{7}+\right)$. MATERIAL. Russia, Dagestan: $1 \sigma^{7}$, [33], Leninsky Komsomol Park, 25.05.2009 (G.Kh.).

COMMENTS. The new record for the Russian fauna. The species was described from South Ukraine and possibly recorded from Georgia [Marusik, Koponen, 2017]. In order to demonstrate that our identification is correct, the male studied is illustrated (Fig. 3).

Dictyna arundinacea (Linnaeus, 1758)

RECORDS. Russia, Dagestan: Yuzhno-Sukhokumsk [Abdurakhmanov et al., 2012].

MATERIAL. Russia, Dagestan: 2 오, [9], 6.07 .2011 (G.Kh.); 1 ㅇ, [9], 2.09.2012 (G.Kh.).

\section{Dictyna ottoi Marusik et Koponen, 2017} Figs 4, 5 . $\left(0^{2}+\right)$

Dictyna ottoi Marusik et Koponen, 2017: 42, figs 1a-d, 2a-g Dictyna ottoi: Esyunin, 2017: 269, figs 20-21 (P).

MATERIAL. Russia, Dagestan: 1 \%, [9], 3.08.2012 (G.Kh.).

COMMENTS. The new record for the Russian fauna. The species is known from Azerbaijan [Marusik, Koponen, 2017] and Georgia [Esyunin, 2017; Otto, Japoshvili, 2018]. The conformation of the epigyne (Figs 4, 5) corresponds that given in the original description [Marusik, Koponen, 2017].

Lathys humilis (Blackwall, 1855)

RECORDS. Russia, Dagestan: Kurush [Abdurakhmanov, Alieva, 2009, 2011].

MATERIAL. Russia, Dagestan: 1 , [33], Leninsky Komsomol Park, 25.05.2009 (G.Kh.). 
Nigma flavescens (Walckenaer, 1830) (G.Kh.).

MATERIAL. Russia, Dagestan: 1 +, [23], 16-18.06.2014

COMMENTS. The new record for the fauna of Dagestan. In the Caucasus, the sepcies is recorded from the south-west part of Krasnodar Territory, Azerbaijan and Armenia [Otto, 2017].

\section{DYSDERIDAE}

Dysdera crocata C.L. Koch, 1838

RECORDS. Russia, DAgESTAN: Makhachkala [Abdurakhmanov, Alieva, 2009, 2011].

MATERIAL. Russia, Dagestan: $1 \sigma^{7}$, [33], Leninsky Komsomol Park, 1.01.2012, (G.Kh.); 1 O, [33], 28.10 .2012 (G.Kh.); o', [33], 1.12.2012 (Galimova).

Dysdera daghestanica Dunin, 1991

RECORDS. Russia, Dagestan: Khuchni [Dunin, 1991a]; Tarki-Tau Mt. [Ponomarev et al., 2008]; Archib, Kondik, Inkhokvari, Khvarshi [Abdurakhmanov, Alieva, 2009, 2011]; Gasha [Abdurakhmanov, Alieva, 2011].

MATERIAl. Russia, Dagestan: $20^{7} \sigma^{x}, 1$, [33], Leninsky Komsomol Park, 27.08.2012 (Galimova); $3 \sigma^{7} \sigma^{7}$, [33], 14.09-1. 12.2012 (Galimova); 1 + , [13], forest, 28.06.2013 (Galimova).

\section{Dysdera ukrainensis Charitonov, 1956}

RECORDS. Russia, DAGESTAN: Bolshie Turali, Makhachkala [Ponomarev, Alieva, 2010; Abdurakhmanov, Alieva, 2011]; Dakhadaevka, Sulak [Ponomarev et al., 2011b]; Inchkhe [Abdurakhmanov et al., 2012]; Sarykum sand dune [Ponomarev et al., 2017b].

MATERIal. Russia, Dagestan: $30^{7} \sigma^{7}$, [18], 10.06.2017 (M.A.); 1 O$^{7}$, [18], tidal saltmarsh, 17.06 .2017 (M.A.); 5 O $^{7}$, [18], 22.04-27.05.2018 (M.A.)

Harpactea modesta Dunin, 1991

RECORDS. Russia, Dagestan: Khuchni [Dunin, 1991b]; Gasha, Tselaygyun [Abdurakhmanov, Alieva, 2011]

MATERIAL. Russia, Dagestan: $1 \sigma^{7}$, [33], Leninsky Komsomol Park, 19.10.2012 (Galimova).

Harpactea spasskyi Dunin, 1992

MATERIAL. Russia, Dagestan: 2 우, [33], Leninsky Komsomol Park, 27.08.2012 (Galimova).

COMMENTS. The new record for the fauna of Dagestan. It is a widespread species in the North Caucasus [Otto, 2017].

\section{ERESIDAE}

Eresus kollari F.W. Rossi, 1846

RECORDS. Russia, Dagestan: Maydanskoe [Abdurakhmanov, Alieva, 2009: Eresus cinnaberinus; 2011]; Bryanskaya Kosa, Tyuleniy Island [Ponomarev et al., 2011a]; Miarso [Ponomarev et al., 2011b]; Yuzhno-Sukhokumsk [Abdurakhmanov et al., 2012]; Chechen Island [Ponomarev, Abdurakhmanov, 2014].

MATerial. Russia, Dagestan: $1 \sigma^{x}$, [19], forest stand, 9.06.2013 (G.Kh.)

\section{GNAPHOSIDAE}

\section{Berlandina saraevi Ponomarev, 2008}

Fig. 6.

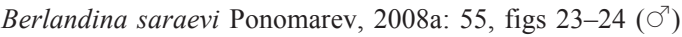
$\left(\sigma^{7}\right)$

Berlandina saraevi: Marusik et al., 2014: 202, figs 69-73

MATERIAl. Russia, Dagestan: $1 \sigma^{\top}$, [19], 04-05.2014 (G.Kh.); 1 O$^{7}$, [19], 5.05.2018 (M.A.).

COMMENTS. The new record for the Russian fauna. The species was described from western Kazakhstan [Ponomarev, 2008a] and to date is known from the type locality only. The palp conformation of the Dagestan specimen (Fig. 6) confirms its belonging to $B$. saraevi.

\section{Callilepis nocturna (Linnaeus, 1758)}

MATERIAL. Russia, Dagestan: 1 +, [9], 6.07.2011 (G.Kh.).

COMMENTS. The new record for the fauna of Dagestan. The species is widespread across the Caucasus [Otto, 2017].

Civizelotes gracilis (Canestrini, 1868)

RECORDS. Russia, DAgEstan: Buynaksk [Ponomarev et al., 2008: Zelotes].

MATERIAL. Russia, Dagestan: $10^{7}$, [19], 05.2013 (G.Kh.).

Drassodes caspius Ponomarev et Tsvetkov, 2006

RECORDS. Russia, Dagestan: Bolshie Turali [Ponomarev, Alieva, 2010; Abdurakhmanov, Alieva, 2011]; Bryanskaya Kosa, Nordovyi Island, Tyuleniy Island, Chechen Island [Ponomarev et al., 2011a]; Chechen Island [Ponomarev, Abdurakhmanov, 2014].

MATERial. Russia, Dagestan: $1 \sigma^{7},[21]$, forest stand, 16.06.2013 (E.I.); 1 O', [19], 04-05.2014 (G.Kh.).

Drassodes dagestanus Ponomarev et Alieva, 2008

RECORDS. Russia, Dagestan: Shalbuzdag Mt. [Ponomarev et al., 2008: Drassodes sp.; Abdurakhmanov, Alieva, 2011: Drassodes sp.]; Archib, Kurush, Khvarshi, Shalbuzdag Mt. [Ponomarev, Alieva, 2010].

MATERIAL. Russia, Dagestan: 2 우, [32], 27.10.2012 (Galimova).

Drassodes lapidosus (Walckenaer, 1802)

RECORDS. Russia, Dagestan: Buynaksk, Dzhaba, Nizhnee Kazanishche, Verkhnee Kazanishche [Ponomarev et al., 2008]; Kondik [Abdurakhmanov, Alieva, 2009, 2011]; Aglobi, Turali, Gasha, Tselyagyun [Abdurakhmanov, Alieva, 2011]; Bryanskaya Kosa, Tyuleniy Island, Chechen Island [Ponomarev et al., 2011a]; Verkhny Gunib, Nizhnee Kazanishche, Novyi Kumukh [Ponomarev et al., 2011b]; Zilbachi [Abdurakhmanov et al., 2012]; Sarykum sand dune, Chechen Island [Ponomarev, Abdurakhmanov, 2014]; Sarykum sand dune [Ponomarev et al., 2017b].

MATERIAL. Russia, Dagestan: $30^{7} \sigma^{7}, 1$, [ [33], 05-3.06.2009 (G.Kh.); $1 \sigma^{7}$, [3], 06.2011 (G.Kh.); 10 O $^{7} \sigma^{2}, 5$ PO, [19], forest stand, 9.06.2013 (G.Kh., E.I.); $5 \sigma^{7} \sigma^{\top}, 5$ OQ , [19], 04-05.2014 (G.Kh.); $2 \sigma^{7} \sigma^{7}$, [21], 20.04 .2013 (E.I.); $2 \sigma^{7} \sigma^{7}, 3$ 우, [21], forest stand, 16.06.2013 (E.I.); 2 O$^{7} \sigma^{7}$, [21], sagebrush, 25.05.2013 (E.I.); 1 ㅇ, [14], gorge, under stones, 18.06.2017 (M.A.); 1 O , [25], 28.05.2017 (M.A.); 1 ㅇ, [8], 28.05.2017 (M.A.); 1 o', [31], 30.04.2017 (M.A.); 1 ㅇ. [7], under stones, 5.06.2017 (M.A.); 1 , [18], tidal saltmarsh, 17.06.2017 (M.A.); $10^{7}$, [24], beach, 5.06. 2013 (E.I.)

Drassodes lutescens (C. L. Koch, 1839)

RECORDS. Russia, Dagestan: Verkhnee Kazanishche [Ponomarev, Khalidov, 2007; Khalidov, Ponomarev, 2008]; Sarykum sand dune [Ponomarev, Abdurakhmanov, 2014; Ponomarev et al., 2017b].

MATERIAL. Russia, Dagestan: 1 ㅇ, [5], meadow, $730 \mathrm{~m}$ a.s.1., 19.06.2017 (M.A.); $1 \sigma^{7}$, [21], 20.04 .2013 (E.I.); $2 \sigma^{7} \sigma^{7}$, 
[21], sagebrush, 5.05.2013 (E.I.); 1 ․, [21], sagebrush, 16.06.2013 (E.I.).

Drassodes pubescens (Thorell, 1856)

RECORDS. Russia, Dagestan: Khvarshi [Abdurakhmanov, Alieva, 2009]; Makhachkala [Abdurakhmanov, Alieva, 2011].

MATERIAL. Russia, Dagestan: 1 + , [11], 16.07.2017 (M.A.).

Drassyllus crimeaensis Kovblyuk, 2003

MATERIAL. Russia, Dagestan: $1 O^{7}$, [21], 20.04.2013 (E.I.); $2 \bigcirc^{7} \sigma^{7}, 1$,, [21], ravine slope with sagebrush, 12.05.2013 (E.I.).

COMMENTS. The new record for the fauna of Dagestan. This species is distributed in the Black Sea region [Nentwig et al., 2018]; in the Caucasus, it is also known from Azerbaijan [Mikhailov, 2013].

\section{Drassyllus praeficus (L. Koch, 1866)}

RECORDS. Russia, Dagestan: Dzhaba, Shalbuzdag Mt., Verkhny Gunib [Ponomarev et al., 2008]; Kondik, Chuvek [Abdurakhmanov, Alieva, 2009, 2011]; Ingishi [Abdurakhmanov et al., 2012]; Sarykum sand dune [Ponomarev, Abdurakhmanov, 2014; Ponomarev et al., 2017b].

MATERIAL. Russia, Dagestan: 1 ơ, [21], 20.04 .2013 (E.I.); $1 \sigma^{7}$, [21], sagebrush, 5.05.2013 (E.I.); $2 \sigma^{7} \sigma^{7}$, [19], 9.05.2015 (G.Kh.).

Drassyllus pusillus (C.L. Koch, 1833)

RECORDS. Russia, Dagestan: Buynaksk, Tsekhok [Ponomarev et al., 2008]; Kondik [Abdurakhmanov, Alieva, 2009, 2011]; Gasha, Makhachkala [Abdurakhmanov, Alieva, 2011]; Tyuleniy Island [Ponomarev et al., 2011a]; Ingishi [Abdurakhmanov et al., 2012].

MATERIAL. Russia, Dagestan: $50^{7} \sigma^{7}, 1$, [23], 5-26.06.2013 (E.I.); 1 +, [24], beach, 5.06 .2013 (E.I.); 1 O , [19], 06.2013 (G.Kh.).

Drassyllus vinealis (Kulczyński in Chyzer et Kulczyński, 1897)

RECORDS. Russia, Dagestan: Nizhnee Kazanishche, Buynaksk [Ponomarev et al., 2008].

MATERIAL. Russia, Dagestan: 1 +, [4], nr. water-power plant, 8.05.2017 (M.A.)

\section{Gnaphosa leporina (L. Koch, 1866)}

RECORDS. Russia, DAGESTAN: Archib, Inkhokvari, Khvarshi [Abdurakhmanov, Alieva, 2009, 2011]; Turali [Abdurakhmanov, Alieva, 2011]; Bryanskaya Kosa, Nordovyi Island, Tyuleniy Island [Ponomarev et al., 2011a]; Sarykum sand dune [Ponomarev, Abdurakhmanov, 2014; Ponomarev et al., 2017b].

MATERIAL. Russia, Dagestan: $1 \sigma^{7}$, [18], 10.06.2017 (M.A.); $1 \sigma^{7},[18]$, tidal saltmarsh, 17.06.2017 (M.A.)

Gnaphosa lucifuga (Walckenaer, 1802)

RECORDS. Russia, Dagestan: Buynaksk, Dzhaba [Ponomarev et al., $2008+$ Gnaphosa sp.]; Chapaevo, Bogatyrevka [Ponomarev et al., 2011b].

MATERIAL. Russia, Dagestan: $10^{\top 7}, 2$ 우, [33], 05.2009 (G.Kh.); 1 + $,[3], 06.2011$ (G.Kh.)

\section{Gnaphosa mongolica Simon, 1895}

RECORDS. Russia, DagESTAn: Bryanskaya Kosa, Tyuleniy Island, Chechen Island [Ponomarev et al., 2011a]; Yuzhno-Sukhokumsk [Abdurakhmanov et al., 2012]; Sarykum sand dune, Chechen Island [Ponomarev, Abdurakhmanov, 2014]; Sarykum sand dune [Ponomarev et al., 2017b].

MATERIAL. Russia, Dagestan: $1 \sigma^{7}$, [19], slope with sagebrush, 9.05.2009 (E.I.) 1992

Gnaphosa steppica Ovtsharenko, Platnick et Song,

RECORDS. Russia, Dagestan: Tselyagyun [Ponomarev, Alieva, 2010; Abdurakhmanov, Alieva, 2011]; Bryanskaya Kosa [Ponomarev et al., 2011a].

MATERIAL. Russia, Dagestan: 1 \%, [19], 04.2009 (G.Kh.); 1 +, [5], meadow, $730 \mathrm{~m}$ a.s.1., 19.06.2017 (M.A.); 2 oq, [29], 20.07.2017 (M.A.).

Haplodrassus caucasius Ponomarev et Dvadnenko, 2013

RECORDS. Russia, Dagestan: Madzhalis [Ponomarev, Dvadnenko, 2013].

MATERIAL. Russia, Dagestan: 2 ㅇ, [23], 26.06.2013 (E.I.).

Haplodrassus dalmatensis (L. Koch, 1866)

RECORDS. Russia, Dagestan: Buynaksk [Ponomarev et al., 2008]; Turali [Abdurakhmanov, Alieva, 2011]; Bryanskaya Kosa, Tyuleniy Island, Chechen Island [Ponomarev et al., 2011a]; Sarykum sand dune, Chechen Island [Ponomarev, Abdurakhmanov, 2014]; Sarykum sand dune [Ponomarev et al., 2017b].

MATERIAL. Russia, Dagestan: $10^{7}$, [21], 25.05 .2013 (E.I.); 1 , [4], nr. water-power plant, 8.05.2017 (M.A.); 1 , [19], slope

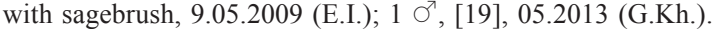

Haplodrassus signifer (C.L. Koch, 1839)

RECORDS. Russia, DAGESTAN: Verkhny Karanay [Ponomarev, Khalidov, 2007; Khalidov, Ponomarev, 2008]; Tsekhok [Ponomarev et al., 2008]; Archib, Kurush, Kondik, Chuvek, Inkhokvari, Khvarshi [Abdurakhmanov, Alieva, 2009, 2011]; Tselyagyun [Abdurakhmanov, Alieva, 2011]; Bryanskaya Kosa [Ponomarev et al., 2011a]; Amsar [Abdurakhmanov et al., 2012]; Chechen Island [Ponomarev, Abdurakhmanov, 2014]; Sarykum sand dune [Ponomarev et al., 2017b].

MATERIAL. Russia, Dagestan: 1 \%, [33], 05.2009 (G.Kh.); 1 $\sigma^{7}$, [21], 20.04.2013 (E.I.); 1 O $^{7}$, [19], 9.05.2015 (G.Kh.).

\section{Kishidaia conspicua (L. Koch, 1866)}

MATERIAL. Russia, Dagestan: 1 \%, [23], 5.06.2013 (E.I.).

COMMENTS. The new record for the fauna of Dagestan. The species is widespread in the Caucasus [Otto, 2017].

Micaria sociabilis Kulczyński in Chyzer et Kulczyński, 1897

MATERIAL. Russia, Dagestan: $1 \mathrm{O}^{7}$, [33], 4.01.2012 (G.Kh.).

COMMENTS. The new record for the fauna of Dagestan. The species is known from southern Europe [Bosmans, Blick, 2000]; in the Caucasus, it is recorded from Azerbaijan [Mikhailov, 2016], and in Russia, from Rostov Area and the Crimea [Ponomarev, Dvadnenko, 2013; Kovblyuk et al., 2016; Mikhailov, 2016].

Nomisia aussereri (L. Koch, 1872)

RECORDS. Russia, Dagestan: Shamkhal [Ponomarev et al., 2011b].

MATERIAL. Russia, Dagestan: 1 , [32], 26.10.2012 (Galimova); 2 +क्, [36], 6.06.2013 (G.Kh.). 
Nomisia conigera (Spassky, 1941) Figs 7-9.

Pterotricha conigera Spassky, 1941: 22, fig. $12\left(\sigma^{7}\right)$. Nomisia conigera: Chatzaki, 2010: 4, figs 6-8 ( ( 7$)$. MATERIAL. Russia, DAgESTAN: 1 \%, [21], 20.04.2013 (E.I.) $8 \sigma^{7} \sigma^{7}, 1$ ㅇ, [21], sagebrush, 5.05-22.06.2013 (E.I.); $12 \sigma^{\top} \sigma^{\top}$, [21], ravine slope with sagebrush, 12.05.2013 (E.I.); 5 O $^{7} \sigma^{7}, 2$ 우, [19], tidal saltmarsh, 22.04-5.05.2018 (M.A.); 2 ○ $\sigma^{\top}$, [19], 12.05.2018 (M.A.).

COMMENTS. The new record for the fauna of Russia. The species was described from Tajikistan [Spassky, 1941] and recorded from Central Asia, Azerbaijan [Mikhailov, 2013] and Turkey [Chatzaki, 2010]. In order to demonstrate that our identification is correct, illustrations of the copulatory organs of the specimens studied are provided (Figs 7-9).

Nomisia exornata (C.L. Koch, 1839)

RECORDS. Russia, Dagestan: Tarki-Tau Mt. [Ponomarev et al., 2008]; Gasha, Turali, Tselyagyun [Abdurakhmanov, Alieva, 2011]; Bryanskaya Kosa, Tyuleniy Island, Chechen Island [Ponomarev et al., 2011a]; Buynaksk [Ponomarev et al., 2011b]; Inchkhe [Abdurakhmanov et al., 2012]; Sarykum sand dune, Chechen Island [Ponomarev, Abdurakhmanov, 2014]; Sarykum sand dune [Ponomarev et al., 2017b]

MATERIAL. Russia, Dagestan: $1 \sigma^{7}$, [22], 9.05 .2012 (G.Kh.); 6 O $^{7} \sigma^{7}, 1$ \%, [19], 04-05.2014, (G.Kh.); 1 O , [19], slope with sagebrush, 9.05.2009 (E.I.); 5 O++ , [19], forest stand, 9.06.2013 (G.Kh.); $3 \bigcirc^{\top} \bigcirc^{7}$, [21], 25.05.2013 (E.I.); 1 \%, [21], forest stand, 16.06.2013 (E.I.); 1 \%, [21], sagebrush, 16.06.2013 (E.I.); $10^{7}, 3$ 우, [18], 10.06.2017 (M.A.); 1 ․ [18], tidal saltmarsh, 17.06.2017 (M.A.); 1 +, [27], 12.06 .2017 (M.A.).

Nomisia ripariensis (O. Pickard-Cambridge, 1872) Fig. 10.

Gnaphosa ripariensis O. Pickard-Cambridge, 1872: 244, pl. 15, fig. 1 ( $\left.\bigcirc^{7}+\right)$.

Nomisia ripariensis: Dunin, 1984: 50, figs 6, 10 ( $\left.\sigma^{7}+\right)$.

Nomisia ripariensis: Chatzaki, 2010: 21, figs 47-50 ( $\left.\sigma^{7}+\right)$.

MATERIAL. Russia, Dagestan: $1 \Im^{\top},[30]$, nr. a pond, 28.05 2017 (M.A.)

COMMENTS. The new record for the fauna of Russia. The species is widespread in the eastern Mediterranean [WSC, 2018]; it was also found in Azerbaijan [Dunin, 1984]. The locality 'Novaya Maka' in Dagestan is the north-easternmost locality for this species. In order to demonstrate that our identification is correct, the copulatory organ of the male studied is illustrated (Fig. 10).

Scotophaeus quadripunctatus (Linnaeus, 1758)

RECORDS. Russia, DAgestan: Tsekhok [Ponomarev et al., 2008]. (E.I.).

MATERIAL. Russia, Dagestan: $10^{\top}$, [24], beach, 5.06.2013

Talanites dunini Platnick et Ovtsharenko, 1991 Fig. 11 .

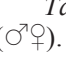

Talanites dunini Platnick et Ovtsharenko, 1991: 117, figs 5-8

Talanites dunini: Levy, 2009: 23, figs 51-52 ().

MATERIAL. Russia, Dagestan: $1 \sigma^{7}$, [19], slope with sagebrush, 9.06.2009 (E.I.); 1 ऽ, [19], 06.2013 (G.Kh.).

COMMENTS. The new record for the fauna of Russia. The species is also known from Azerbaijan, Turkmenistan
[Platnick, Ovtsharenko, 1991] and Israel [Levy, 2009]. The locality 'Sarykum sand dune' in Dagestan is the northernmost locality for this species. The male of $T$. dunini was illustrated in the original descrioption only [Platnick, Ovtsharenko, 1991]. For comparison, an illustration of the male palp of the specimen studied is provided (Fig. 11).

\section{Talanites cf. dunini Platnick et Ovtsharenko, 1991} Fig. 12.

MATERIAL. Russia, Dagestan: $2 \sigma^{\top} \sigma^{7},[21]$, sagebrush, 16.06.2013 (E.I.)

COMMENTS. Based on the palpal conformation (Fig. 12), this species is close to $T$. dunini, but differs in the embolic structure and larger size. The taxonomic status of this species will be considered in more details elsewhere.

Talanites fagei Spassky, 1938

RECORDS. Russia, Dagestan: Makhachkala [Ponomarev, Alieva, 2010; Abdurakhmanov, Alieva, 2011]; Bryanskaya Kosa, Tyuleniy Island [Ponomarev et al., 2011a]; Sarykum sand dune [Ponomarev et al., 2017b].

MATERIAL. Russia, Dagestan: $1 \sigma^{7}$, [24], beach, 5.06.2013 (E.I.)

Trachyzelotes adriaticus (Caporiacco, 1951)

RECORDS. Russia, Dagestan: Bryanskaya Kosa, Nordovyi Island, Tyuleniy Island [Ponomarev et al., 2011a].

MATERIAL. Russia, Dagestan: $10^{7}$, [18], 10.06.2017 (M.A.); $1 \mathrm{O}^{7}$, [18], 27.05 .2018 (M.A.).

Trachyzelotes jaxartensis (Kroneberg, 1875)

MATERIAL. Russia, Dagestan: 1 \%, [31], 30.04.2017 (M.A.).

COMMENTS. The new record for the fauna of Dagestan. On the territory of Russia, the species is known from Astrakhan Area [Kulczyński, 1901] and Kalmykia [Platnick, Murphy, 1984]; in Transcaucasia, it was recorded from Azerbaijan [Khasayeva, Huseynov, 2017].

Trachyzelotes malkini Platnick et Murphy, 1984

RECORDS. Russia, Dagestan: Bolshie Turali, Makhachkala [Ponomarev, Alieva, 2010; Abdurakhmanov, Alieva, 2011]; Bryanskaya Kosa, Tyuleniy Island [Ponomarev et al., 2011a]; Tatayurt [Abdurakhmanov et al., 2012]; Sarykum sand dune [Ponomarev et al., 2017b].

MATERIAL. Russia, Dagestan: 1 \%, [36], 6.06.2013 (G.Kh.); $1 \mathrm{O}^{7}$, [4], nr. water-power plant, 8.05.2017, (M.A.); 1 ․, [18], 10.06.2017 (M.A.); 1 \%, [18], tidal saltmarsh, 17.06.2017 (M.A.).

Trachyzelotes manytchensis Ponomarev et Tsvetkov, 2006

MATERIAL. Russia, Dagestan: $10^{7}$, [18], 10.06.2017 (M.A.).

COMMENTS. The new record for the fauna of Dagestan. The species was described from Rostov Area [Ponomarev, Tsvetkov, 2006] and was also reported from Stavropol Territory [Ponomarev et al., 2017a].

Trachyzelotes pedestris (C.L. Koch, 1837)

RECORDS. Russia, Dagestan: Makhachkala [Ponomarev, Alieva, 2010; Abdurakhmanov, Alieva, 2011].

MATERIAL. Russia, Dagestan: $3 \sigma^{7} \sigma^{\top}, 1$,, [23], 5-26.06.2013 (E.I.); 1 O', 3 +क, [33], 1.05-16.06.2018 (M.A., G.Kh.).

Zelotes atrocaeruleus (Simon, 1878)

RECORDS. Russia, DAgESTAN: Bryanskaya Kosa [Ponomarev et al., 2011a]. 

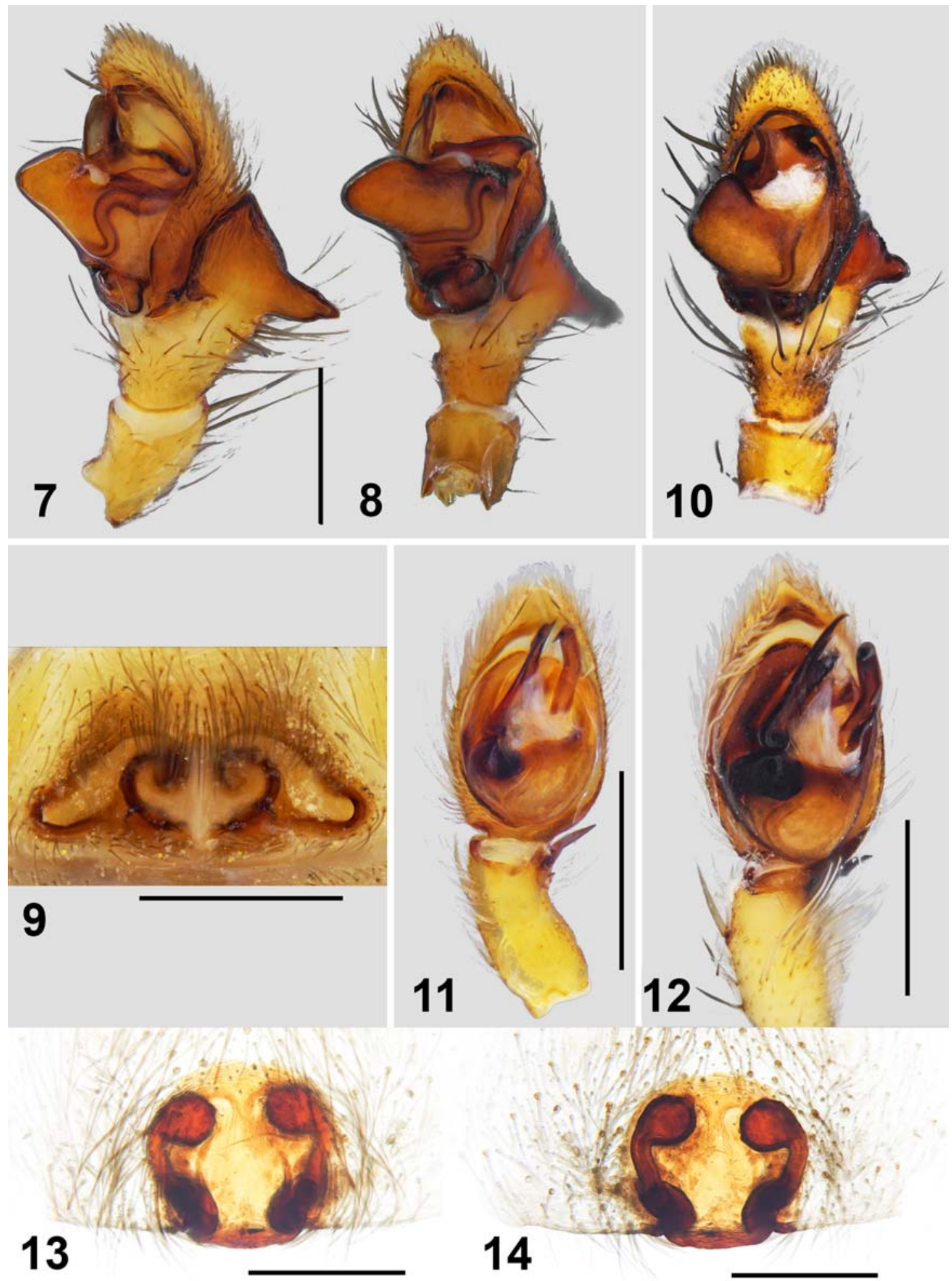

Figs 7-14. Copulatory organs of Nomisia conigera (Spassky, 1941) (7-9), N. ripariensis (O. Pickard-Cambridge, 1872) (10), Talanites dunini Platnick et Ovtsharenko, 1991 (11), Talanites cf. dunini (12), Alopecosa pentheri (Nosek, 1905) (13, 14): 7 — male palp, retrolateral view; 8, 10 - male palp, ventro-retrolateral view; 9, 13 - epigyne, ventral view; 11-12 - male palp, ventral view; 14 spermathecae, dorsal view. Scale bar: $0.5 \mathrm{~mm}$.

Рис. 7-14. Копулятивные органы Nomisia conigera (Spassky, 1941) (7-9), N. ripariensis (O. Pickard-Cambridge, 1872) (10), Talanites dunini Platnick et Ovtsharenko, 1991 (11), Talanites cf. dunini (12), Alopecosa pentheri (Nosek, 1905) (13, 14): 7 — пальпа самца ретролатерально; 8, 10 - пальпа самца вентро-ретролатерально; 9, 13 - эпигина, вид снизу; 11-12 - пальпа самца, вид снизу; 14 - сперматека, вид сверху. Масштаб: 0,5 мм. 
MATERIAL. Russia, Dagestan: $1 \sigma^{\top}$, [18], tidal saltmarsh, 17.06.2017 (M.A.).

Zelotes khostensis Kovblyuk et Ponomarev, 2008 MATERIAL. Russia, Dagestan: 1 ․ [9], 3.08 .2012 (G.Kh.).

COMMENTS. The new record for the fauna of Dagestan, but widespread in the Caucasus [Kovblyuk, Ponomarev, 2008; Otto, 2017]. It was also recorded from Italy [Pantini, Mazzoleni, 2018].

Zelotes longipes (L. Koch, 1866)

RECORDS. Russia, Dagestan: Yuzhno-Sukhokumsk, Tarumovka [Abdurakhmanov et al., 2012]; Sarykum sand dune, Chechen Island [Ponomarev, Abdurakhmanov, 2014]; Sarykum sand dune [Ponomarev et al., 2017b].

MATERIAL. Russia, Dagestan: 1 ㅇ, [34], 1.10 .2012 (G.Kh.); 1 , , [19], 9.03.2013 (G.Kh.); 1 , [19], 04-05.2014 (G.Kh.); 1 \% , [19], 14.05.2014, (G.Kh.); 1 +, [14], gorge, under stones, 18.06.2017 (M.A.).

Zelotes orenburgensis Tuneva et Esyunin, 2003

RECORDS. Russia, DAgESTAN: Makhachkala [Abdurakhmanov et al., 2012].

MATERIAL. Russia, Dagestan: $10^{7}$, [18], 10.06.2017 (M.A.).

Zelotes petrensis (C.L. Koch, 1839)

RECORDS. Russia, Dagestan: Gasha, Makhachkala [Ponomarev, Alieva, 2010; Abdurakhmanov, Alieva, 2011]; Sarykum sand dune [Ponomarev et al., 2017b].

MATERIAL. RusSia, DaGestan: $1+$, [14], gorge, under stones, 18.06.2017 (M.A.).

Zelotes segrex (Simon, 1878)

RECORDS. Russia, Dagestan: Tyuleniy Island [Ponomarev et al., 2011a]; Yuzhno-Sukhokumsk [Abdurakhmanov et al., 2012]; Sarykum sand dune [Ponomarev, Abdurakhmanov, 2014].

MATERIAL. Russia, Dagestan: $3 \sigma^{7} \sigma^{7},[21]$, ravine slope with sagebrush, 12.05.2013 (E.I.); $1 \mathrm{O}^{7}$, [21], sagebrush, 16.06.2013 (E.I.).

\section{LINYPHIIDAE}

Agyneta rurestris (C.L. Koch, 1836)

RECORDS. Russia, Dagestan: Dzhaba [Ponomarev et al., 2008].

MATERIAL. Russia, Dagestan: 1 +, [31], 30.04.2017 (M.A.).

Agyneta saaristoi Tanasevitch, 2000

MATERIAL. Russia, Dagestan: 1 + , [19], 14.05 .2014 (G.Kh.).

COMMENTS. The new record for the fauna of Dagestan; a species distributed in the south of the European part of Russia and Kazakhstan [Nennwig et al., 2018].

Frontinellina frutetorum (C.L. Koch, 1834)

RECORDS. Russia, Dagestan: Gasha, Tselyagyun [Ponomarev, Alieva, 2010; Abdurakhmanov, Alieva, 2011]; Nizhnee Kazanishche [Ponomarev et al., 2011b].

MATERIAL. Russia, Dagestan: 1 \%, [19], 14.05.2014 (G.Kh.); 2 우, [5], meadow, $730 \mathrm{~m}$ a.s.1., 19.06.2017 (M.A.); 1 \%, [8], 28.05.2017 (M.A.).

Gongylidium rufipes (Linnaeus, 1758)

MATERIAL. Russia, Dagestan: 1 \%, [9], 2.08 .2012 (G.Kh.).

COMMENTS. The new record for the fauna of Dagestan. In the Caucasus, it is also known from Karachay-
Cherkesia [Tanasevitch, 1990] and North Ossetia [Ponomarev, Komarov, 2013].

Ipa terrenus (L. Koch, 1879)

RECORDS. Russia, Dagestan: Garakh [Tanasevitch, 1990: Lepthyphantes quadrimaculatus].

MATERIAL. Russia, Dagestan: 1 , [28], juniper grove, 13.08.2012 (E.I.).

Linyphia triangularis (Clerck, 1758)

RECORDS. Russia, Dagestan: Makhachkala, Tselyagyun [Ponomarev, Alieva, 2010; Abdurakhmanov, Alieva, 2011]; Nizhnee Kazanishche [Ponomarev et al., 2011b]; Sulak [Ponomarev, Abdurakhmanov, 2014].

MATERIAL. Russia, Dagestan: $1 \sigma^{7}$, [9], 3.08 .2012 (G.Kh.).

Microlinyphia pusilla (Sundevall, 1830)

RECORDS. Russia, Dagestan: Erpeli, Verkhnee Kazanishche, Verkhny Karanay [Ponomarev, Khalidov, 2007; Khalidov, Ponomarev, 2008]; Bogatyrevka [Ponomarev et al., 2011b]; Yuzhno-Sukhokumsk [Abdurakhmanov et al., 2012].

MATERIAL. Russia, Dagestan: 1 \%, [9], 27.07.2012 (G.Kh.).

Neriene emphana (Walckenaer, 1841)

RECORDS. Russia, DAgESTAN: Erpeli, Verkhny Karanay [Ponomarev, Khalidov, 2007; Khalidov, Ponomarev, 2008]. MATERIAL. Russia, Dagestan: 1 + [9], 6.07.2011 (G.Kh.); 1 +, [9], 27.07.2012 (G.Kh.).

Oedothorax apicatus (Blackwall, 1850)

MATERIAL. Russia, Dagestan: 1 +, [3], 11.06.2013 (Alibekova, Abdusalimova)

COMMENTS. The new record for the fauna of Dagestan. This species is widespread across the Caucasus [Otto, 2017].

Tenuiphantes mengei (Kulczyński, 1887)

RECORDS. Russia, Dagestan: Gunib, Degva [Tanasevitch, 1987].

MATERIAL. Russia, Dagestan: 1 +, [9], 6.07 .2011 (G.Kh.).

\section{LIOCRANIDAE}

Agroeca cuprea Menge, 1873

RECORDS. Russia, Dagestan: Madzhalis [Ponomarev, Alieva, 2010; Abdurakhmanov, Alieva, 2011]; Sarykum sand dune [Ponomarev et al., 2017b].

MATERIAL. Russia, Dagestan: 1 \% , [13], forest, 17.07.2013 (Galimova)

\section{LYCOSIDAE}

Allohogna singoriensis (Laxmann, 1770)

RECORDS. Russia, Dagestan: Buynaksk Distr. [Ponomarev et al., 2008]; Makhachkala [Abdurakhmanov, Alieva, 2009, 2011]; Nordovyi Island, Tyuleniy Island [Ponomarev et al., 2011a]; Shamkhal [Ponomarev et al., 2011b]; Chechen Island [Ponomarev, Abdurakhmanov, 2014].

MATERIAL. Russia, Dagestan: 1 +, [18], tidal saltmarsh, 1.07.2017 (M.A.).

Alopecosa albofasciata (Brullé, 1832)

RECORDS. Russia, Dagestan: Tarki-Tau Mt. [Ponomarev et al., 2008]; Tselyagyun [Abdurakhmanov, Alieva, 
2011]; Inchkhe [Abdurakhmanov et al., 2012]; Sarykum sand dune [Ponomarev et al., 2017b].

MATERIAL. Russia, Dagestan: $1 \sigma^{7}, 1$ o, [22], 9.05 .2012 (G.Kh.).

Alopecosa cronebergi (Thorell, 1875)

RECORDS. Russia, DAgEstan: Derbent [Thorell, 1875: sub Tarentula c.]; Bryanskaya Kosa, Tyuleniy Island, Chechen Island [Ponomarev et al., 2011a]; Sarykum sand dune [Ponomarev et al., 2017b].

MATERIAL. Russia, Dagestan: 1 ㅇ, [21], sagebrush, 5.05.2013 (E.I.); 1 +, [18], 19.05.2018 (M.A.).

\section{Alopecosa cursor (Hahn, 1831)}

RECORDS. Russia, Dagestan: Bolshie Turali, Tselyagyun [Ponomarev, Alieva, 2010; Abdurakhmanov, Alieva, 2011]; Bryanskaya Kosa, Tyuleniy Island, Chechen Island [Ponomarev et al., 2011a]; Novolakskoe, Sarykum sand dune [Ponomarev et al., 2011b]; Inchkhe [Abdurakhmanov et al., 2012]; Chechen Island [Ponomarev, Abdurakhmanov, 2014]; Sarykum sand dune [Ponomarev et al., 2017b].

MATERIAL. Russia, Dagestan: 1 \%, [6], 15.04 .2012 (G.Kh.) 1 Oे $^{\top} 1$ ㅇ, [19], 04.2013 (Z. Shavlukov); 1 +, [19], 04-05.2014 (G.Kh.); 5 우, [21], sagebrush, 5.05.2013 (E.I.)

\section{Alopecosa pentheri (Nosek, 1905)} Figs 13,14

Pardosa pentheri Nosek, 1905: 141, figs 21, 24 ( $\left.\sigma^{7}+\right)$

Alopecosa cursor pentheri: Lugetti, Tongiorgi, 1969: 55, figs $15 \mathrm{f}-\mathrm{c}\left(\bigcirc^{7}\right)$.

$(+)$.

Alopecosa insignis: Lugetti, Tongiorgi, 1969: 57, figs 15d-e

Alopecosa pentheri: Dunin, 1984: 47, fig. $3(+)$.

Alopecosa pentheri: Thaler et al., 2000: 1073, figs 3-4, 42 , 46-47 ( $\left.\sigma^{7}+\right)$

Alopecosa pentheri: Marusik et al., 2018: 358: figs 1F-K, 2B, 3C-D, 4C-D, 5G-L, 6I-J, 7H-K, 8B-C ( ${ }^{7}+$ ).

MATERIAL. Russia, Dagestan: 3 +o, [21], ravine slope with sagebrush, 12.05.2013 (E.I.); 1 ㅇ, [21], sagebrush, 16.06.2013 (E.I.).

COMMENTS. The new record for the fauna of Dagestan. This species is widespread in south-eastern Europe, Azerbaijan, Turkey [Nentwig et al., 2018]. On the territory of Russia, it is known from the Crimea [Mikhailov, 2013]. In order to confirm that our identification is correct, illustrations of the specimens studied are provided (Figs 13, 14).

Alopecosa trabalis (Clerck, 1758)

RECORDS. Russia, DAGESTAN: Gasha, Madzhalis [Ponomarev, Alieva, 2010; Abdurakhmanov, Alieva, 2011].

MATERIAL. Russia, Dagestan: 1 \%, [10], 8.07.2013 (A.A. Omarov, I.M. Ushanov).

Arctosa cinerea (Fabricius, 1777)

RECORDS. Russia, Dagestan: Gasha [Ponomarev, Alieva, 2010; Abdurakhmanov, Alieva, 2011]; Shamkhal [Ponomarev et al., 2011b].

MATERIAL. Russia, Dagestan: 1 + , [6], 9.04 .2017 (E.I.).

Arctosa leopardus (Sundevall, 1832)

RECORDS. Russia, Dagestan: Bolshie Turali, Makhachkala [Ponomarev, Alieva, 2010; Abdurakhmanov, Alieva, 2011]; Bryanskaya Kosa, Nordovyi Island, Tyuleniy Island [Ponomarev et al., 2011a]; Sarykum sand dune [Ponomarev et al., 2017b].

MATERIAL. Russia, Dagestan: $1 \sigma^{7}$, [24], beach, 5.06.2013 (E.I.); $1 \sigma^{7}$, [27], 12.06 .2017 (M.A.).
Arctosa personata (L. Koch, 1872)

RECORDS. Russia, Dagestan: Tarki-Tau Mt. [Ponomarev et al., 2008]; Sarykum sand dune [Ponomarev et al., 2017b].

MATERIAL. Russia, Dagestan: 1 ○ , [19], 04-05.2014 (G.Kh.).

Arctosa tbilisiensis Mcheidze, 1947

RECORDS. Russia, Dagestan: Bolshie Turali, Gasha, Makhachkala [Ponomarev, Alieva, 2010; Abdurakhmanov, Alieva, 2011]; Bryanskaya Kosa [Ponomarev et al., 2011a].

MATERIAL. RusSia, Dagestan: $2 \sigma^{7} \sigma^{7}$, [9], 6.07-10.08.2011 (G.Kh.); 1 O$^{7}$, [19], 06.2013 (G.Kh.); 1 ㅇ, [24], beach, 5.06.2013 (E.I.)

Aulonia albimana (Walckenaer, 1805)

RECORDS. Russia, Dagestan: Talgi Canyon [Ponomarev et al., 2011b].

MATERIAL. Russia, Dagestan: 1 \%, [13], 5.08.2012 (Galimova).

Bogdocosa kronebergi (Andreeva, 1976)

RECORDS. Russia, Dagestan: Bryanskaya Kosa, Tyuleniy Island, Chechen Island [Ponomarev et al., 2011a: sub. $B$. baskuntchakensis]; Sarykum sand dune, Chechen Island [Ponomarev, Abdurakhmanov, 2014: sub. B. baskuntchakensis]; Sarykum sand dune [Ponomarev et al., 2017b: sub. B. baskuntchakensis; Esyunin, Ponomarev, 2018].

MATERIAL. Russia, Dagestan: $1 \sigma^{7}, 2$ O+, [19], 9.06.2013 (E.I.); $1 \mathrm{O}^{\top}, 1$, 1 , [19], forest stand, 9.06.2013 (G.Kh.); $4 \mathrm{O}^{\top} \mathrm{O}^{\top}, 3$ 우, [19], 04-05.2014 (G.Kh.). 2003

Evippa apsheronica Marusik, Guseinov et Koponen,

RECORDS. Russia, Dagestan: Nordovyi Island, Tyuleniy Island, Chechen Island [Ponomarev et al., 2011a]; Chechen Island [Ponomarev, Abdurakhmanov, 2014].

MATERIAL. Russia, Dagestan: $2 \sigma^{7} \sigma^{7}, 2$ 우, [3], 06.2011 (Gasanov); $2 \bigcirc^{7} \sigma^{7}, 1$, [3], 24.06 .2012 (G.Kh.).

Hogna radiata (Latreille, 1817)

RECORDS. Russia, Dagestan: Buynaksk Distr., Kaspiysk, Karabudakhkent, Gumi, Izberbash [Ponomarev et al., 2008]; Aglobi [Abdurakhmanov, Alieva, 2009]; Makhachkala, Kondik [Abdurakhmanov, Alieva, 2009, 2011]; Izberbash, Tselyagyun [Abdurakhmanov, Alieva, 2011]; Erpeli, Buynaksk, Verkhnee Kazanishche, Nizhnee Kazanishche, Dakhadaevka, Novokuli, Bogatyrevka [Ponomarev et al., 2011b]; Sarykum sand dune [Ponomarev, Abdurakhmanov, 2014].

MATERIAL. Russia, Dagestan: $1 \sigma^{7}$, [3], 06.2011 (G.Kh.); 1 +, [19], 13.10.2013 (G.Kh.); $1 \mathrm{O}^{7}$, [18], tidal saltmarsh, 1.07.2017 (M.A.).

Lycosa cf. praegrandis C.L. Koch, 1836

RECORDS. Russia, Dagestan: Sarykum sand dune [Ponomarev et al., 2017b: Lycosa praegrandis].

MATERIAL. Russia, Dagestan: 1 + [38], 20.06.2013 (G.Kh.).

Mustelicosa dimidiata (Thorell, 1875)

RECORDS. Russia, Dagestan: Verkhny Karanay [Ponomarev, Khalidov, 2007; Khalidov, Ponomarev, 2008]; Aimaki, Karabudakhkent, Khunzakh [Ponomarev et al., 2008]; Kondik, Chuvek, Tsuduk, Maydanskoe [Abdurakhmanov, Alieva, 2009, 2011]; Turali [Abdurakhmanov, Alieva, 2011]; Bryanskaya Kosa, Tyuleniy Island, Chechen Island [Pono- 
marev et al., 2011a]; Verkhny Gunib [Ponomarev et al., 2011b]; Yuzhno-Sukhokumsk [Abdurakhmanov et al., 2012]; Sarykum sand dune, Chechen Island [Ponomarev, Abdurakhmanov, 2014].

MATERIAL Russia, Dagestan: 1 \% [3], 06.2011 (G.Kh.); 2 $\sigma^{7} \sigma^{7}$, [19], forest stand, 9.06.2013 (G.Kh.); $3 \sigma^{7} \sigma^{7}$, [19], 9.06.2013 (E.I.); $1 \sigma^{7}$, [19], 04-05.2014 (G.Kh.); $11 \sigma^{7} \sigma^{7}$, [21], forest stand, 16.06.2013 (E.I.); $1 \mathrm{O}^{\mathrm{T}}$, [18], tidal saltmarsh, 1.07.2017 (M.A.); 1 + [14], gorge, under stones, 18.06.2017 (M.A.); 1 + , [29], 20.07. 2017 (M.A.); 1 ㅇ, [7], under stones, 5.06.2017 (M.A.); 1 O', [18], 10.06.2017 (M.A.).

Pardosa agrestis (Westring, 1861)

RECORDS. Russia, Dagestan: Erpeli, Verkhnee Kazanishche, Verkhny Karanay [Ponomarev, Khalidov, 2007; Khalidov, Ponomarev, 2008]; Buynaksk, Verkhny Gunib, Verkhnee Kazanishche, Dzhaba, Dubki, Nizhnee Kazanishche, Khunzakh, Khuchni [Ponomarev et al., 2008]; Archib, Kurush, Maydanskoe, Makhachkala, Kondik, Chuvek, Khvarni, Tsuduk [Abdurakhmanov, Alieva, 2009, 2011]; Turali, Tselyagyun [Abdurakhmanov, Alieva, 2011]; Bryanskaya Kosa [Ponomarev et al., 2011a]; Verkhny Gunib, Nizhnee Kazanishche, Novyi Kumukh, Novolakskoe, Sulak, Talgi Canyon [Ponomarev et al., 2011b]; Tarumovka [Abdurakhmanov et al., 2012].

MATERIAL. Russia, Dagestan: $1 \sigma^{7}$, [22], 9.05 .2012 (G.Kh.); $1 \mathrm{O}^{7}$, [9], 27.07.2012 (G.Kh.); 4 우, [11], 16.07.2017, (M.A.); 1 , [19], 05.2013 (G.Kh.).

Pardosa amentata (Clerck, 1758)

RECORDS. Russia, Dagestan: Buynaksk [Ponomarev et al., 2008].

MATERIAL. Russia, Dagestan: $10^{7}, 2$ 우, [1], $1500 \mathrm{~m}$ a.s.1., 13.04.2013 (Galimova).

Pardosa caucasica Ovtsharenko, 1979

RECORDS. Russia, Dagestan: Erpeli, Verkhnee Kazanishche, Verkhny Karanay [Ponomarev, Khalidov, 2007; Khalidov, Ponomarev, 2008]; Buynaksk, Verkhny Gunib, Tsekhok [Ponomarev et al., 2008]; Kondik [Abdurakhmanov, Alieva, 2009, 2011]; Talgi Canyon [Ponomarev et al., 2011b]

MATERIAL. Russia, Dagestan: 1 + , [9], 6.07.2011 (G.Kh.).

Pardosa jaikensis Ponomarev, 2007

RECORDS. Russia, DAGESTAN: Verkhny Karanay [Ponomarev, Khalidov, 2007; Khalidov, Ponomarev, 2008: in both cases sub P. italica]; Verkhny Dzhengutai, Dubki [Ponomarev et al., 2008: sub P. italica and Pardosa sp. 2]; Turali [Abdurakhmanov, Alieva, 2011: sub. P. italica]; Bryanskaya Kosa, Nordovyi Island [Ponomarev et al., 2011a].

MATERIAL. RusSia, DAGESTAN: 1 + , [19], tidal saltmarsh, 05. 2013 (G.Kh.)

Pardosa lugubris (Walckenaer, 1802)

RECORDS. Russia, Dagestan: Gasha [Abdurakhmanov, Alieva, 2011].

MATERIAL. Russia, Dagestan: 1 \%, [9], 6.07.2011 (G.Kh.).

Pardosa nebulosa (Thorell, 1872)

RECORDS. Russia, DagESTAN: Derbent [Thorell, 1875]; Makhachkala [Abdurakhmanov, Alieva, 2009, 2011]; Gasha [Abdurakhmanov, Alieva, 2011]; Bryanskaya Kosa [Ponomarev et al., 2011a]; Sulak [Ponomarev et al., 2011b; Ponomarev, Abdurakhmanov, 2014]; Sarykum sand dune [Ponomarev et al., 2017b].

MATERIAL. Russia, Dagestan: 1 9, [19], 04-05.2014 (G.Kh.); $12 \sigma^{7} \sigma^{7}, 4$ 우, [3], 06.2013 (Abdullaeva, Magomedova); $16 \sigma^{7} \sigma^{7}$, 5 우, [3], 11.06.2013 (Alibekova, Abdusalimova).
Pardosa pontica (Thorell, 1875)

RECORDS. Russia, DAgEstan: Buynaksk Distr. [Ponomarev et al., 2008]; Irganay [Abdurakhmanov, Alieva, 2009, 2011]; Gasha, Makhachkala [Abdurakhmanov, Alieva, 2011]; Bryanskaya Kosa, Tyuleniy Island [Ponomarev et al., 2011a]; Sulak [Ponomarev et al., 2011b].

MATERIAL. Russia, Dagestan: 1 \%, [3], 24.06.2012 (G.Kh.); 2 우, [27], 12.06.2017 (M.A.).

\section{Pardosa proxima (C.L. Koch, 1847)}

RECORDS. Russia, Dagestan: Kondik [Abdurakhmanov, Alieva, 2009, 2011]; Gasha, Tselyagyun [Abdurakhmanov, Alieva, 2011].

MATERIAL. Russia, Dagestan: $1 \bigcirc^{\top}$, [13], 28.04.2012 (Galimova).

\section{Pardosa schenkeli Lessert, 1904}

RECORDS. Russia, Dagestan: Kurush, Shalbuzdag Mt. [Buchar, Thaler, 1998].

MATERIAL. Russia, Dagestan: 1 O', [7], 5.06.2017 (M.A.).

Pardosa tasevi Buchar, 1968

RECORDS. Russia, DAgESTAN: Erpeli [Ponomarev, Khalidov, 2007; Khalidov, Ponomarev, 2008].

MATERial. Russia, Dagestan: $1 \sigma^{7}$, [5], meadow, $730 \mathrm{~m}$ a.s.1., 19.06.2017 (M.A.).

Pardosa tatarica (Thorell, 1875)

RECORDS. Russia, Dagestan: Kondik, Chuvek, Tsuduk, Inkhokvari, Khonokh, Khvarshi [Abdurakhmanov, Alieva, 2009, 2011].

MATERIAL. Russia, Dagestan: 1 \% , [3], 06.2011 (G.Kh.).

Piratula latitans (Blackwall, 1841)

RECORDS. Russia, Dagestan: Buynaksk [Ponomarev et al., 2008: Pirata]; Nizhnee Kazanishche [Ponomarev et al., 2011b: Pirata]; Makhachkala [Abdurakhmanov, Alieva, 2011: Pirata].

MATERIAL. Russia, DAgESTAN: 1 ○', [24], beach, 5.06.2013 (E.I.); $2 \sigma^{\top} \sigma^{\top}$, [21], 06.2013 (G.Kh.).

Trochosa ruricola (De Geer, 1778)

RECORDS. Russia, DAGESTAN: Erpeli [Ponomarev, Khalidov, 2007; Khalidov, Ponomarev, 2008]; Buynaksk, Verkhnee Kazanishche, Nizhnee Kazanishche, Tsekhok [Ponomarev et al., 2008]; Kondik, Chuvek, Tsuduk [Abdurakhmanov, Alieva, 2009, 2011]; Gasha, Tselyagyun [Abdurakhmanov, Alieva, 2011]; Bryanskaya Kosa, Nordovyi Island [Ponomarev et al., 2011a]; Nizhnee Kazanishche, Buynaksk, Novyi Kumukh [Ponomarev et al., 2011b]; Gunib [Abdurakhmanov et al., 2012]; Makhachkala [Ponomarev, Abdurakhmanov, 2014]; Sarykum sand dune [Ponomarev et al., 2017b].

MATERIAl. Russia, Dagestan: 1 9, [3], 06.2011 (G.Kh.); 3 $\mathrm{O}^{7} \sigma^{7}$, [3], 11.06.2013 (Alibekova, Abdusalimova); $22 \mathrm{O}^{7} \mathrm{O}^{7}, 7$ 우, [23], 5-26.06.2013 (E.I.); 2 ऽ $^{\top} \sigma^{7}$, [24], beach, 5.06.2013 (E.I.); 1 $\sigma^{7}$, [13], forest, 17.07.2013 (Galimova).

Trochosa terricola Thorell, 1856

RECORDS. Russia, Dagestan: Erpeli, Verkhnee Kazanishche, Verkhny Karanay [Ponomarev, Khalidov, 2007; Khalidov, Ponomarev, 2008]; Tarki-Tau Mt. [Ponomarev et al., 2008]; Makhachkala, Kondik, Chuvek [Abdurakhmanov, Alieva, 2009, 2011]; Bogatyrevka [Ponomarev et al., 2011b]; Amsar [Abdurakhmanov et al., 2012].

MATERIAL. Russia, Dagestan: $3 \sigma^{7} \sigma^{7}$, [23], 5.06 .2013 (E.I.); 1 †, [23], 16-18.06.2014, (G.Kh.); 1 ठ , [13], forest, 28.06.2013 (Galimova). 
Xerolycosa nemoralis (Westring, 1861)

RECORDS. Russia, DagESTAN: Verkhnee Kazanishche, Verkhny Karanay [Ponomarev, Khalidov, 2007; Khalidov, Ponomarev, 2008]; Tsekhok [Ponomarev et al., 2008].

MATERIAL. Russia, Dagestan: 1 ㅇ, [9], 31.07.2012 (G.Kh.).

\section{MIMETIDAE}

Mimetus laevigatus (Keyserling, 1863)

RECORDS. Russia, Dagestan: Gasha [Ponomarev, Alieva, 2010; Abdurakhmanov, Alieva, 2011]; Gunib [Ponomarev et al., 2011b].

MATERIAL. Russia, Dagestan: 1 \%, [19], 14.05 .2014 (G.Kh.); 1 + , [8], 28.05.2017 (M.A.)

\section{MITURGIDAE}

Zora spinimana (Sundevall, 1832)

RECORDS. Russia, Dagestan: Erpeli, Verkhnee Kazanishche [Ponomarev, Khalidov, 2007; Khalidov, Ponomarev, 2008]; Talgi Canyon [Ponomarev et al., 2011b].

MATERIAL. RusSia, Dagestan: 1 + , [20], 3.08.2013 (Galimova).

\section{OXYOPIDAE}

Oxyopes globifer Simon, 1876

RECORDS. Russia, Dagestan: Bryanskaya Kosa, Chechen Island [Ponomarev et al., 2011a].

MATERIAL. Russia, Dagestan: $1 \sigma^{7}$, [3], 06.2013 (Abdullaeva, Magomedova); $1 \sigma^{7}$, [21], sagebrush, 22.06.2013 (E.I.); 2 O $^{7} \mathrm{O}^{7}$,

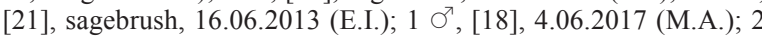
$\sigma^{7} \sigma^{7},[27], 12.06 .2017$ (M.A.).

\section{Oxyopes heterophthalmus (Latreille, 1804)}

RECORDS. Russia, DAGESTAN: Erpeli [Ponomarev, Khalidov, 2007; Khalidov, Ponomarev, 2008]; Maydanskoe [Abdurakhmanov, Alieva, 2009, 2011]; Gasha [Abdurakhmanov, Alieva, 2011]; Bryanskaya Kosa, Tyuleniy Island [Ponomarev et al., 2011a]; Bogatyrevka, Nizhnee Kazanishche [Ponomarev et al., 2011b]; Sarykum sand dune [Ponomarev, Abdurakhmanov, 2014; Ponomarev et al., 2017b].

MATERIAL. Russia, Dagestan: 1 9, [9], 6.08 .2012 (G.Kh.); 1 +, [21], 25.05.2013 (E.I.); 1 + , [21], sagebrush, 16.06.2013 (E.I.) $1 \mathrm{O}^{2}, 2$ 우, [35], 6.06.2013 (G.Kh.); 3 우, [36], 6.06.2013 (G.Kh.); 1 o , 1 \%, [3], 06.2013 (Abdullaeva, Magomedova); 1 o , 2 ᄋᄋ, [37], field, 11.06.2013 (G.Kh.); 4 O $^{7} \sigma^{7}, 5$ 우, [8], 28.05.2017 (M.A.); $1 \sigma^{7}$, [30], nr. a pond, 28.05 .2017 (M.A.); 2 Oᄋ, [5], meadow, $730 \mathrm{~m}$ a.s.1., 19.06.2017 (M.A.); $3 \mathrm{O}^{7} \mathrm{O}^{7}$, , [25], 28.05.2017 (M.A.); 1 O, 3 우, [27], 12.06.2017 (M.A.)

Oxyopes lineatus Latreille, 1806

RECORDS. Russia, Dagestan: Buynaksk Distr. [Ponomarev et al., 2008]; Maydanskoe [Abdurakhmanov, Alieva, 2009, 2011]; Gasha [Abdurakhmanov, Alieva, 2011]; Bryanskaya Kosa [Ponomarev et al., 2011a]; Nizhnee Kazanishche, Buynaksk, Novolakskoe [Ponomarev et al., 2011b]; Yuzhno-Sukhokumsk [Abdurakhmanov et al., 2012]; Sarykum sand dune, Chechen Island [Ponomarev, Abdurakhmanov, 2014]; Sarykum sand dune [Ponomarev et al., 2017b].

MATERIAL. Russia, Dagestan: 1 + , [19], 20.06 .2009 (G.Kh.); $1 O^{7},[19], 06.2009$ (S. Gamzatova); $2 \mathrm{O}^{7} \sigma^{7}, 2$ 우, [19], 2.07.2013 (P.I. Tagirova, M.M. Shakhmanova); 5 O $^{7} \sigma^{7}, 9$ 우, [23], 25.06.2017 (M.A.); 3 O $^{7} \sigma^{7}$, [27], 12.06 .2017 (M.A.)

\section{PHILODROMIDAE}

Philodromus cespitum Walckenaer, 1826

RECORDS. Russia, Dagestan: Maydanskoe [Abdurakhmanov, Alieva, 2009, 2011]; Nizhnee Kazanishche [Ponomarev et al., 2011b].
MATERIAL. Russia, Dagestan: 1 ㅇ, [9], 6.07.2011 (G.Kh.); 1 †, [27], 12.06.2017 (M.A.)

Philodromus poecilus (Thorell, 1872)

MATERIAL. Russia, Dagestan: 1 \%, [23], 16-18.06.2014 (G.Kh.)

COMMENTS. The new record for the fauna of Dagestan. This species is widespread in the Palaearctic Region [WSC, 2018].

Pulchellodromus ruficapillus (Simon, 1885)

RECORDS. Russia, Dagestan: Sarykum sand dune [Ponomarev et al., 2017b].

MATERIAL. Russia, Dagestan: $1 \sigma^{7}$, [3], 06.2013 (Abdullaeva, Magomedova); 3 O $^{7} \sigma^{7}, 1$ ㅇ, [8], 28.05.2017 (M.A.); 2 O $^{7} \sigma^{7}, 1$ +, [27], 12.06.2017 (M.A.)

COMMENTS. The species is rare and local in Dagestan, but it is widespread in the Mediterranean and was also recorded from Kazakhstan [Nentwig et al., 2018]. In Russia, it was also found in the Crimea [Kastrygina, Kovblyuk, 2014], Cis-Azov Area [Ponomarev et al., 2016] and the northern part of Stavropol Territory [Ponomarev et al., 2017a].

Rhysodromus histrio (Latreille, 1819)

RECORDS. Russia, Dagestan: Bryanskaya Kosa [Ponomarev et al., 2011a: Philodromus histrio].

MATERIAL. Russia, Dagestan: 1 + [31], 30.04 .2017 (M.A.); 3 웅, [8], 28.05.2017 (M.A.)

Rhysodromus timidus (Szita et Logunov, 2008)

Figs 15-19. $79\left(0^{7}\right)$

Philodromus timidus Szita et Logunov, 2008: 53, figs 33-35,

Philodromus timidus: Logunov et al., 2011: 235, figs 1-8 (○ౌo). Rhysodromus timidus: Kastrygina, Kovblyuk, 2016: 284.

RECORDS. Russia, Dagestan: Chechen Island [Ponomarev, Dvadnenko, 2013: Philodromus].

MATERIAL. Russia, Dagestan: 1 \%, [27], 12.06.2017 (M.A.); 1 ․ [18], 19.05.2018 (M.A.)

COMMENTS. In Russia, this species has been found in Dagestan only [Ponomarev, Dvadnenko, 2013]; it is also known from Kazakhstan and Pakistan [Logunov et al., 2011].

The material studied contains only two females. We have provisionally assigned them to $R$. timidus, as both have the transverse row of dense bristles in front of their spinnerets (Figs 15, 16), which is typical of this species. However, the epigynal conformation of these females (Figs 17-19) is somewhat different from that provided by Logunov et al. [2011]. Males are required to prove/reject the current identification.

Thanatus atratus Simon, 1875

RECORDS. Russia, Dagestan: Kurush [Abdurakhmanov, Alieva, 2009, 2011]; Nizhnee Kazanishche, Sulak [Ponomarev et al., 2011b].

MATERIAL. Russia, Dagestan: $10^{7}$, [29], 20.07.2017 (M.A.).

Thanatus imbecillus L. Koch, 1878

RECORDS. Russia, Dagestan: Tarki-Tau Mt. [Ponomarev et al., 2008]; Bryanskaya Kosa [Ponomarev et al., 2011a]; Inchkhe [Abdurakhmanov et al., 2012].

MATERIAL. Russia, Dagestan: $1 \sigma^{7}$, [4], nr. water-power plant, 8.05.2017 (M.A.); 1 ○', [22], 9.05.2012 (G.Kh.); 1 ㅇ, [21], sagebrush, 5.05.2013 (E.I.); 8 O $^{2} \sigma^{2}$, [21], ravine slope with sagebrush, 12.05.2013 (E.I.). 

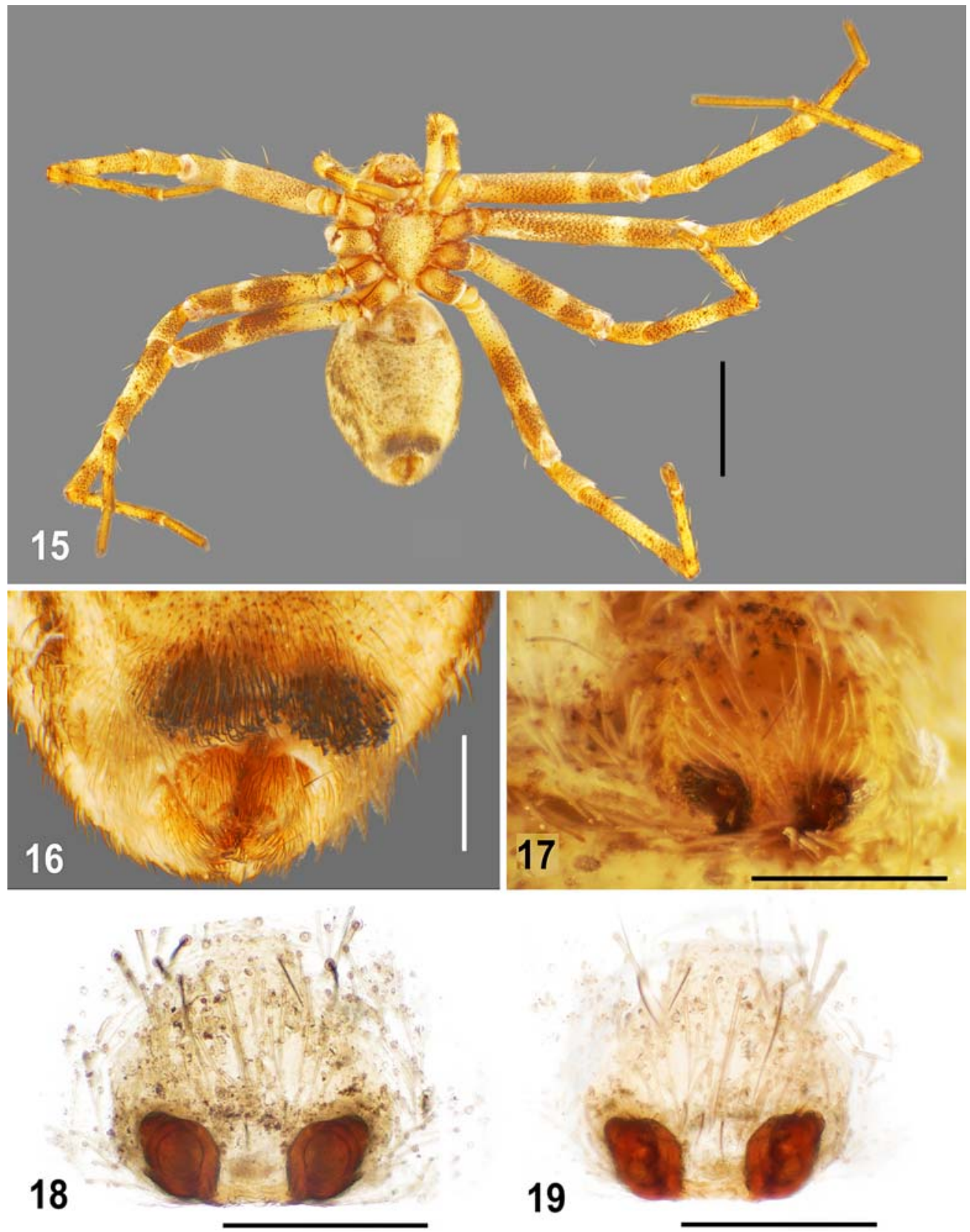

Figs 15-19. Habitus and copulatory organs of Rhysodromus timidus Szita et Logunov, 2008: 15 - female, ventral view; 16 — caudal part of abdomen, ventral view; 17, 18 - epigyne, ventral view; 19 - spermathecae, dorsal view. Scale bars: (15) $0.5 \mathrm{~mm},(16-19) 0.25 \mathrm{~mm}$.

Рис. 15-19. Габитус и копулятивные органы Rhysodromus timidus Szita et Logunov, 2008: 15 - самка, вид снизу; 16 каудальная часть брюшка, вид снизу; 17, 18 - эпигина, вид снизу; 19 - сперматека, вид сверху. Масштаб: (15) 0,5 мм, (16-19) 0,25 мм.

Thanatus kitabensis Charitonov, 1946

RECORDS. Russia, Dagestan: Chechen Island [Ponomarev, Dvadnenko, 2012: Thanatus ubsunurensis, misidentification]; Sarykum sand dune [Ponomarev et al., 2017b].

MATERIAL. Russia, Dagestan: $10^{7}$, [13], 24.07.2012 (Galimova).

Thanatus vulgaris Simon, 1870

RECORDS. Russia, Dagestan: Dzhaba, Nizhny Dzhengutai [Ponomarev et al., 2008]; Bryanskaya Kosa, Nordovyi
Island, Tyuleniy Island, Chechen Island [Ponomarev et al., 2011 a]; Yuzhno-Sukhokumsk [Abdurakhmanov et al., 2012]; Chechen Island [Ponomarev, Abdurakhmanov, 2014]; Sarykum sand dune [Ponomarev et al., 2017b].

MATERIAL. Russia, Dagestan: 1 \%, [19], 20.06 .2009 (G.Kh.); 1 , [19], 06.2009 (S. Gamzatova); 1 , [3], 06.2013 (Abdullaeva, Magomedova); $1 \mathrm{O}^{\text {T. }}$, [18], tidal saltmarsh, 17.06 .2017 (M.A.).

Tibellus maritimus (Menge, 1875)

MATERIAL. Russia, Dagestan: $1 \sigma^{7}$, [27], 12.06 .2017 (M.A.). 

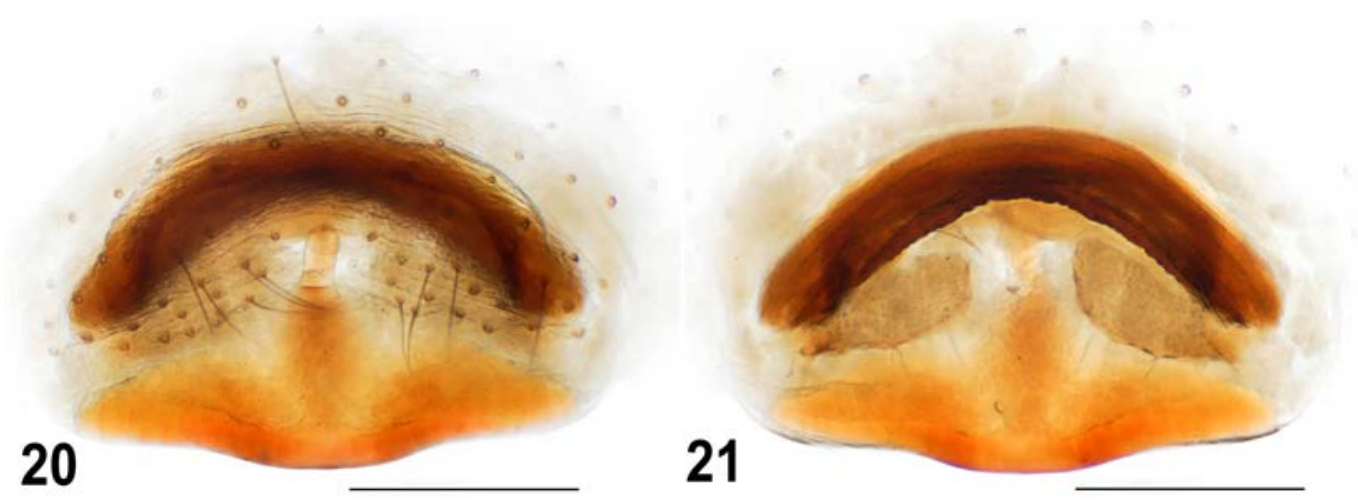

Figs 20-21. Copulatory organs of Pholcus sogdianae Brignoli, 1978: 20 - epigyne, ventral view; 21 - spermathecae, dorsal view. Scale bars: $0.25 \mathrm{~mm}$.

Рис. 20-21. Копулятивные органы Pholcus sogdianae Brignoli, 1978: 20 - эпигина, вид снизу; 21 - сперматека, вид сверху. Масштаб: 0,25 мм.

COMMENTS. The new record for the fauna of Dagestan. This species is widespread in the Palaearctic [WSC, 2018].

Tibellus oblongus (Walckenaer, 1802)

RECORDS. Russia, Dagestan: Erpeli [Ponomarev, Khalidov, 2007; Khalidov, Ponomarev, 2008]; Khunzakh [Ponomarev et al., 2008]; Bryanskaya Kosa, Tyuleniy Island [Ponomarev et al., 2011a]; Nizhnee Kazanishche, Novyi Kumukh [Ponomarev et al., 2011b]; Chechen Island [Ponomarev, Abdurakhmanov, 2014].

MATERIAL. Russia, Dagestan: $1 \sigma^{7}$, [37], field, 11.06.2013 (G.Kh.); 1 + , [26], 11.06.2017 (G.Kh.); 1 O', [27], 12.06.2017 (M.A.).

\section{PHOLCIDAE}

Pholcus crassipalpis Spassky, 1937

RECORDS. Russia, Dagestan: Derbent [Huber, 2011]. MATERIAL. Russia, Dagestan: 1 + [34], 10.04.2012 (G. Kh.)

Pholcus sogdianae Brignoli, 1978

Figs 20, 21.

Pholcus fagei Spassky, 1940: 357, figs 6-8 ( (フㅇ).

Pholcus sogdianae Brignoli, 1978: 489.

Pholcus sogdianae: Huber, 2011: 348, figs 1643, 1663-1664, 1693-1694, 1697-1698, 2192-2193 (O'O).

MATERIAL. Russia, Dagestan: $19,[14]$, gorge, under stones, 18.06.2017 (M.A.).

COMMENTS. The new record for the fauna of Russia. This species is distributed in Central Asia [WSC, 2018]. In order to confirm that our identification is correct, illustrations of the female studied are provided (Figs 20, 21).

\section{PHRUROLITHIDAE}

Phrurolithus pullatus Kulczyński in Chyzer et Kulczyński, 1897

MATERIAL. Russia, Dagestan: 1 , [21], ravine slope with sagebrush, 12.05.2013 (E.I.)

COMMENTS. The new record for the fauna of Dagestan. The species is rare in the Caucasus, known from its north-western regions [Ponomarev, Volkova, 2013] and Azerbaijan [Otto, 2017] only.

\section{PISAURIDAE}

Pisaura mirabilis (Clerck, 1758)

RECORDS. Russia, Dagestan: Chechen Island [Ponomarev, Abdurakhmanov, 2014].

MATERIAL. Russia, Dagestan: 4 우, [2], 23.06 .2011 (G.Kh.); 1, , [17], 6.06.2017 (E.I.).

\section{Pisaura novicia (L. Koch, 1878)}

RECORDS. Russia, Dagestan: Sarykum sand dune, Chechen Island [Ponomarev, Abdurakhmanov, 2014]; Sarykum sand dune [Ponomarev et al., 2017b].

MATERIAL. Russia, Dagestan: 1 \%, [33], 3.06 .2009 (N.M. Gasanova); 1 , [24], beach, 5.06 .2013 (E.I.); 1 ○', 3 우, [23], 26.06.2013 (E.I.); 1 \%, [23], 16-18.06.2014 (G.Kh.); 1 O', [23], 1.04.2017 (M.A.).

\section{SALTICIDAE}

Aelurillus concolor Kulczyński, 1901

Fig. 23 .

Aelurillus concolor Kulczyński, 1901: 349, pl. 13, fig. 18 (ㅇ). $\left(\bigcirc^{\top}+\right)$.

Aelurillus concolor: Wesołowska, 1996: 21, figs 2A-C, 3F-C $A$
$\left(\sigma^{\top}+\right)$.

Aelurillus concolor: Azarkina, Mirshamsi, 2014: 83, figs 2-33

MATERIAL. Russia, Dagestan: $1 \sigma^{7},[21]$, ravine slope with sagebrush, 12.05.2013 (E.I.); 1 ऽ, [21], sagebrush, 22.06.2013 (E.I.); $2 \sigma^{7} \sigma^{7}$, [19], tidal saltmarsh, 5-12.05.2018 (M.A.); 1 ๙", [18], 5.05.2018 (M.A.)

COMMENTS. The new record for the fauna of Russia. This species is knonw from Greece, Turkey, Iran, Azerbaijan, Georgia, Kazakhstan, and Turkmenistan [Mikhailov, 2013; Azarkina, Mirshamsi, 2014]. The absence of the tibial buldge on the male palp of the specimens studied (Fig. 23) is evidence that our identification is correct.

Aelurillus lutosus (Tyschchenko, 1965)

Fig. 22. $\left(\mathrm{O}^{\top}+\right)$

Aelurillus lutosus: Logunov, Marusik, 2000: 265, figs 7-11

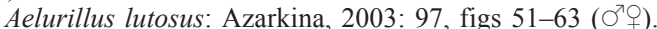

MATERIAL. Russia, Dagestan: 1 \%, [6], 15.04.2012 (G.Kh.). 

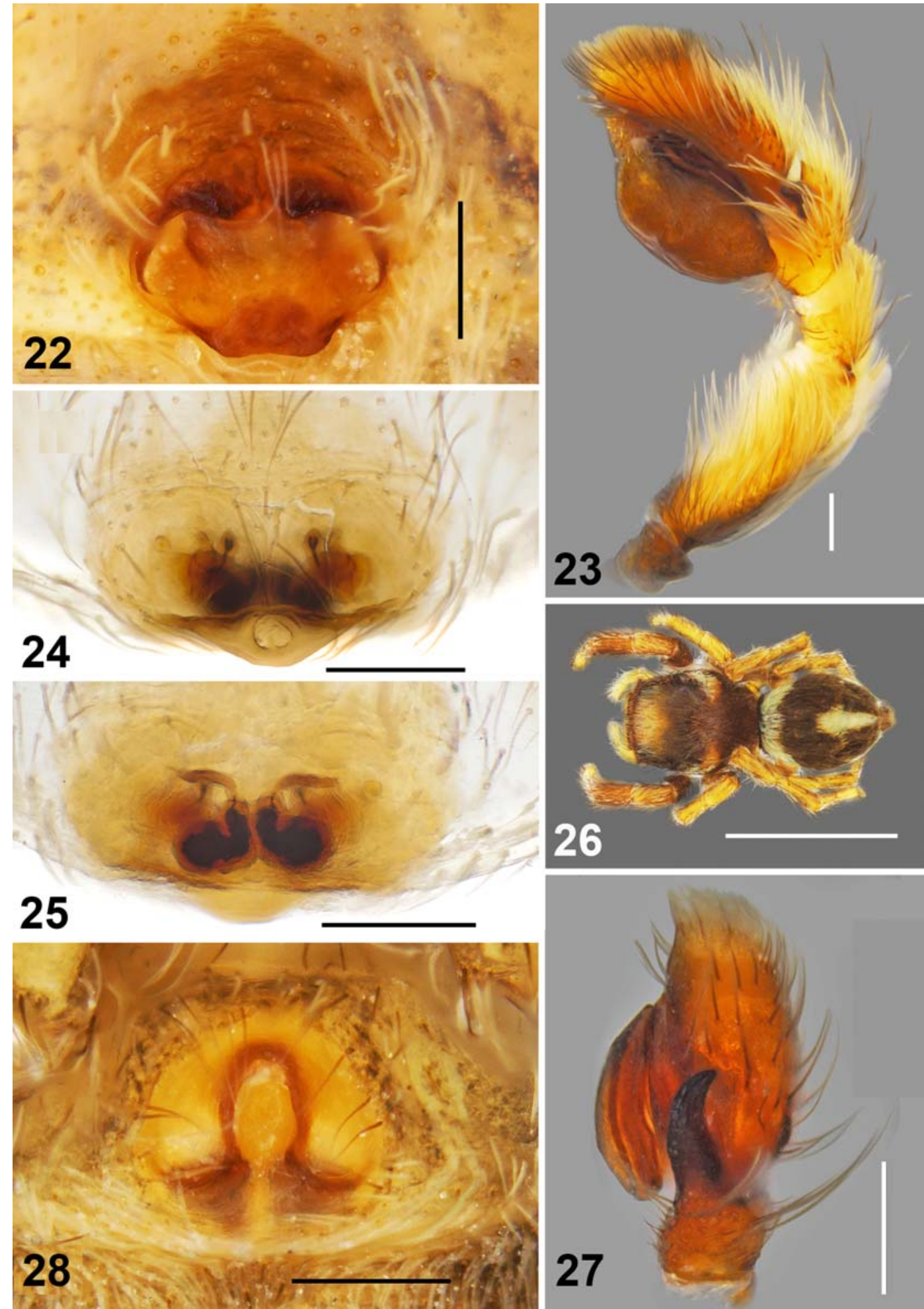

Figs 22-28. Copulatory organs and habitus of Aelurillus lutosus (Tyschchenko, 1965) (22), A. concolor Kulczyñski, 1901 (23), Chinattus caucasicus Logunov, 1999 (24, 25) and Pellenes geniculatus (Simon, 1868) (26-28): 22, 24, 28 - epigyne, ventral view; 23, 27 - male palp, lateral view; 25 - spermathecae, dorsal view; 26 - female, dorsal view. Scale bars: $(22,23,27,28) 0.25 \mathrm{~mm},(24,25)$ $0.2 \mathrm{~mm},(26) 2.5 \mathrm{~mm}$.

Рис. 22-28. Копулятивные органы и габитус Aelurillus lutosus (Tyschchenko, 1965) (22), A. concolor Kulczycski, 1901 (23), Chinattus caucasicus Logunov, 1999 (24, 25) и Pellenes geniculatus (Simon, 1868) (26-28): 22, 24, 28 - эпигина, вид снизу; 23, 27 пальпа самца, вид сбоку; 25 - сперматека, вид сверху; 26 - самка, вид сверху. Масштаб: $(22,23,27,28) 0,25$ мм, $(24,25) 0,2$ мм, (26) 2,5 мм. 
COMMENTS. The new record for the fauna of Dagestan. The species is known from Kazakhstan and northern Kirghizia [Azarkina, 2003]; in Russia, it was recently reported from Astrakhan Area [Ponomarev, Alekseev, 2018]. In order to confirm that our identification is correct, illustration of the epigyne is provided (Fig. 22).

Aelurillus v-insignitus (Clerck, 1758)

RECORDS. Russia, Dagestan: Sulak [Ponomarev et al., 2011b]; Sarykum sand dune, Agrakhan Peninsula, Sulak [Ponomarev, Abdurakhmanov, 2014]; Sarykum sand dune [Ponomarev et al., 2017b].

MATERIAL. Russia, Dagestan: $10^{7}$, [34], 1.10 .2012 (G.Kh.);

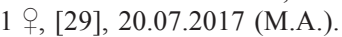

Calositticus caricis (Westring, 1861)

MATERIAL. Russia, Dagestan: 1 q, [23], 16-18 .06 .2014 (G.Kh.).

COMMENTS. The new record for the fauna of Dagestan. This species is widespread in Europe; in the Caucasus, it was recorded from Georgia [Mikhailov, 2013; Nentwig et al., 2018]. 1997)

Calositticus inexpectus (Logunov et Kronestedt,

MATERIal. Russia, Dagestan: $1 \bigcirc^{\top}, 1$ +, [27], 12.06 .2017 (M.A.).

COMMENTS. The new record for the fauna of Dagestan. This species is widespread in western and southern Europe, and Central Asia [Nentwig et al., 2018; WSC, 2018]; in Russia, it was recorded from the Cis-Azov Sea region [Ponomarev et al., 2016].

Chalcoscirtus tanasevichi Marusik, 1991

MATERIAL. Russia, Dagestan: 1 + , [29], 20.07.2017 (M.A.)

COMMENTS. The new record for the fauna of Russia. It was recorded from Turkey, Armenia, Azerbaijan, Kazakhstan and Kyrgyzstan [Mikhailov, 2013; Nentwig et al., 2018].

Chinattus caucasicus Logunov, 1999

Figs 24, 25.

Chinattus caucasicus Logunov, 1999: 146, figs 40-47 ( $\bigcirc^{7}+$ ).

MATERIAL. Russia, Dagestan: 2 우, [23], 16-18.06.2014 (G.Kh.); 1 ․, [23], 1-25.05.2018 (M.A.).

COMMENTS. The new record for the fauna of Russia. This species was known from Azerbaijan, Armenia, Georgia and Iran [Logunov, 1999]. As the species remains known from the original description only, illustrations of the epigyne of the females studied are provided (Figs 24, 25).

Euophrys frontalis (Walckenaer, 1802)

MATERIAL. RusSia, DAGESTAN: 1 + [14], gorge, under stones, 18.06.2017 (M.A.); 1 O', [4], nr. water-power plant, 8.05.2017 (M.A.).

COMMENTS. The new record for the fauna of Dagestan. This species is widespread in the Palaearctic Region [WSC, 2018].

Evarcha arcuata (Clerck, 1758)

RECORDS. Russia, DAgestan: Kasumkent [Logunov, Guseinov, 2002]; Erpeli [Ponomarev, Khalidov, 2007; Khalidov, Ponomarev, 2008]; Verkhny Gunib [Ponomarev et al., 2008]; Gasha [Abdurakhmanov, Alieva, 2011]; Yuzhno-Sukhokumsk [Abdurakhmanov et al., 2012].

MATERIAL. Russia, Dagestan: $1 \sigma^{\top}, 2$ 우, [9], 3-6.08.2012 (G.Kh.); $1 \sigma^{7}$, [3], 06.2013 (Abdullaeva, Magomedova); $1 \odot^{7}$, [24], beach, 5.06.2013 (E.I.); $1 \mathrm{O}^{7}$, [5], meadow, $730 \mathrm{~m}$ a.s.1., 19.06.2017 (M.A.); 1 O', [23], 25.06 .2017 (M.A.).

Evarcha michailovi Logunov, 1992

RECORDS. Russia, Dagestan: Derbent [Logunov, Guseinov, 2002].

MATERial. Russia, Dagestan: 2 우 [5], meadow, $730 \mathrm{~m}$ a.s.1., 19.06.2017 (M.A.)

Heliophanus auratus C.L. Koch, 1835

RECORDS. Russia, DagESTAN: Bryanskaya Kosa, Tyuleniy Island [Ponomarev et al., 2011a]; Chechen Island [Ponomarev, Abdurakhmanov, 2014]; Sarykum sand dune [Ponomarev et al., 2017b].

MATERIal. Russia, Dagestan: 1 +, [19], 2.07.2013 (P.I. Tagirova, M.M. Shakhmanova); 1 , , [27], 12.06.2017 (M.A.); 1 ㅇ, [24], beach, 5.06.2013 (E.I.).

Heliophanus cupreus (Walckenaer, 1802)

RECORDS. Russia, DAGESTAN: Southern Dagestan [Rakov, Logunov, 1997: fig. 35]; Gasha [Abdurakhmanov, Alieva, 2011]; Sarykum sand dune [Ponomarev et al., 2017b].

MATERIAL. Russia, Dagestan: $1 \mathrm{O}^{7}$, [9], 2.08.2012 (G.Kh.).

Heliophanus dunini Rakov et Logunov, 1997

RECORDS. Russia, Dagestan: Bryanskaya Kosa, Tyuleniy Island, Chechen Island [Ponomarev et al., 2011a]; Yuzhno-Sukhokumsk [Abdurakhmanov et al., 2012]; Sulak, Chechen Island [Ponomarev, Abdurakhmanov, 2014].

MATERIAL. Russia, Dagestan: 1 \%, [19], 06.2009 (G.Kh.); 1

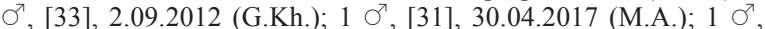
[18], 4.06.2017 (M.A.); 1 O', [27], 12.06.2017 (M.A.).

Heliophanus mordax (O. Pickard-Cambridge, 1872)

RECORDS. Russia, Dagestan: Kisilyurt [Rakov, Logunov, 1997]; Derbent [Logunov, Guseinov, 2002]; Makhachkala [Abdurakhmanov, Alieva, 2011]; Sarykum sand dune [Ponomarev et al., 2017b].

MATERIAL. Russia, Dagestan: $1 \sigma^{7}$, [19], slope with sagebrush, 9.05.2009 (E.I.); 1 丁 $^{7}$, [19], 14.05.2014 (G.Kh.); 3 o $^{7} \sigma^{7}, 6$ 우, [35], 6.06.2013 (G.Kh.); 11 우, [36], 6.06.2013 (G.Kh.).

Mogrus antoninus Andreeva, 1976

RECORDS. Russia, Dagestan: Sarykum sand dune [Ponomarev et al., 2017b].

MATERIAL. Russia, Dagestan: $10^{7}$, [27], 12.06.2017 (M.A.).

Mogrus neglectus (Simon, 1868)

RECORDS. Russia, Dagestan: Derbent [Logunov, Guseinov, 2002]; Chechen Island [Ponomarev, Abdurakhmanov, 2014]; Sarykum sand dune [Ponomarev et al., 2017b].

MATERIAL. Russia, Dagestan: 2 우, [3], 06.2013 (Abdullaeva, Magomedova); 1 ㅇ, [10], 8.07.2013 (A.A. Omarov, I.M. Ushanov); 1 \%, [19], 14.05.2014 (G.Kh.); 1 +, [31], 30.04 .2017 (M.A.); $2 \sigma^{7} \sigma^{7}, 1$ ․ [8], 28.05.2017 (M.A.); $1 \Im^{7}$, [30], nr. a pond, 28. 05.2017 (M.A.).

\section{Pellenes geniculatus (Simon, 1868)} Figs 26-28.

Attus geniculatus Simon, 1868: $49\left(\mathrm{O}^{7}\right)$.

Pellenes geniculatus: Dunin, 1984: 52, fig. 14 ().

Pellenes geniculatus: Logunov et al., 1999: 126, figs 5, 131$153\left(\sigma^{2}+\right)$.

MATERIAL. Russia, Dagestan: 1 , [16], 10.04.2012 (E.I.); 2 $\sigma^{\top} \sigma^{\top},[21]$, sagebrush, 5.05 .2013 (E.I.); $3 \sigma^{\top} \sigma^{7}$, [21], ravine slope with sagebrush, 12.05 .2013 (E.I.); 1 \%, [19], 14.05.2014 (G.Kh.); $3 \circlearrowleft^{7} \sigma^{7}$, [19], tidal saltmarsh, 12.05.2018 (M.A.). 
COMMENTS. The new record for the fauna of Dagestan. In Russia, the species was previously known from the Crimea only [Mikhailov, 2013]. The specimens recorded from the territory of Russia have not been illustrated to date; this is why our illustrations are provided (Fig 26-28).

Pellenes nigrociliatus (Simon in L. Koch, 1875)

RECORDS. Russia, Dagestan: Yuzhno-Sukhokumsk [Abdurakhmanov et al., 2012].

MATERIAL. Russia, Dagestan: $1 \sigma^{7},[21]$, ravine slope with sagebrush, 12.05.2013 (E.I.).

\section{Pellenes seriatus (Thorell, 1875)}

RECORDS. Russia, Dagestan: Bryanskaya Kosa [Ponomarev et al., 2011a]; Sarykum sand dune [Ponomarev et al., 2017b].

MATERIAL. Russia, Dagestan: 1 \% [3], 06.2011 (Gasanov); 1 P, [36], 6.06.2013 (G.Kh.). 1999

Pellenes turkmenicus Logunov, Marusik et Rakov,

RECORDS. Russia, Dagestan: Derbent [Logunov, Guseinov, 2002]; Bolshie Turali [Abdurakhmanov, Alieva, 2011]; Bryanskaya Kosa [Ponomarev et al., 2011a].

MATERIAL. Russia, DAgestan: 1 + , [8], 28.05.2017 (M.A.).

Philaeus chrysops (Poda, 1761)

RECORDS. Russia, Dagestan: Derbent [Logunov, Guseinov, 2002]; Gunib, Dubki, Tarki-Tau Mt., Khunzakh [Ponomarev et al., 2008]; Makhachkala, Irganay [Abdurakhmanov, Alieva, 2009, 2011]; Buynaksk, Karabudakhkent, Sarykum sand dune [Ponomarev et al., 2011b]; YuzhnoSukhokumsk [Abdurakhmanov et al., 2012]; Sarykum sand dune, Chechen Island [Ponomarev, Abdurakhmanov, 2014]; Sarykum sand dune [Ponomarev et al., 2017b].

MATERIAL. Russia, Dagestan: 1 o", [3], 06.2011 (G.Kh.); 1 †, [3], 06.2013 (Abdullaeva, Magomedova); $10^{7}$, [36], 6.06.2013 (G.Kh.); $10^{7}$, [35], 6.06.2013 (G.Kh.); $20^{7} \sigma^{7}, 2$ 우, [4], nr. waterpower plant, 8.05.2017 (M.A.); $2 \sigma^{7} \sigma^{\top}, 2$ 우, [29], 20.07.2017 (M.A.).

\section{Phlegra lineata (C.L. Koch, 1846)}

Figs 29, 30 .

Euophrys lineata C.L. Koch, 1846: 43, fig. $1303\left(\sigma^{7}\right)$.

Phlegra lineata: Metzner, 1999: 70, figs 35a-1 ( ( 7 ( $)$.

Phlegra lineata: Prószyński, 2003: 137, figs 555-561 ( $\sigma^{7}+$ ).

MATERIAL. Russia, Dagestan: 1 \%, [9], 31.07 .2012 (G.Kh.); 1 , [19], tidal saltmarsh, 3.05.2018 (M.A.).

COMMENTS. The new record for the fauna of Russia. The species was known from southern Europe, Turkey and Syria [WSC, 2018]. The locality in Dagestan (Dylym, Sarykum sand dune) are the north-easternmost records of the species range. The general appearance and the conformation of the epigyne of the females studied (Figs 29, 30) are evidence that our identification is correct.

Pseudeuophrys erratica (Walckenaer, 1825)

RECORDS. Russia, Dagestan: Degva [Logunov, 1998]; Verkhnee Kazanishche, Verkhny Karanay [Ponomarev, Khalidov, 2007; Khalidov, Ponomarev, 2008]; Madzhalis [Abdurakhmanov, Alieva, 2011]; Talgi Canyon [Ponomarev et al., 2011b].

MATERIAL. Russia, Dagestan: $10^{7}$, [9], 6.07.2011 (G.Kh.); 1 , [23], 5.06.2013 (E.I.).
Pseudicius encarpatus (Walckenaer, 1802)

RECORDS. RusSia, DAgestan: Aglobi [Abdurakhmanov, Alieva, 2009, 2011]

MATERIAL. Russia, Dagestan: $1 \sigma^{7}$, [3], 06.2013 (Abdullaeva, Magomedova); 1 ○ै, [23], 16-18.06.2014 (G.Kh.).

Pseudomogrus vittatus (Thorell, 1875)

RECORDS. Russia, Dagestan: Bryanskaya Kosa, Tyuleniy Island [Ponomarev et al., 2011a: sub. Yllenus v.]; Yuzhno-Sukhokumsk [Abdurakhmanov et al., 2012: sub. Yllenus v.].

MATERIAL. Russia, Dagestan: 1 , [3], 06.2013 (Abdullaeva, Magomedova).

Salticus zebraneus (C.L. Koch, 1837)

MATERIAL. Russia, Dagestan: $10^{7}$, [9], 31.07 .2012 (G.Kh.).

COMMENTS. The new record for the fauna of Dagestan. In the Caucasus, the species was recorded from Krasnodar Territory [Ponomarev, Volkova, 2013], Adygea [Ponomarev et al., 2012] and North Ossetia [Ponomarev, Komarov, 2013].

Yllenus zyuzini Logunov et Marusik, 2003 Figs 31, 32.

Yllenus zyuzini Logunov et Marusik, 2003: 116, figs 45, 53, 55, 69-70, 394-405 (O'T).

MATERIAL. Russia, Dagestan: 2 ○ $\sigma^{7}$, [19], 27.04.2013 (G.Kh.); $1 \sigma^{7}$, [19], 19-22.09.2013 (G.Kh.).

COMMENTS. The new record for the fauna of Russia. This species is known from Kazakhstan and Turkmenistan [Logunov, Marusik, 2003]; it was also recorded from the northern Caspian region, in the shore of Inder Lake [Ponomarev, 2008b]. Hitherto, the species was illustrated in the original description only. In order to demonstrate that our identification is correct, the palp of the males studied is illustrated (Figs 31, 32).

\section{SCYTODIDAE}

Scytodes thoracica (Latreille, 1802)

RECORDS. Russia, Dagestan: Verkhny Dzhengutai, Nizhnee Kazanishche [Ponomarev et al., 2008]; Tselyagyun [Abdurakhmanov, Alieva, 2011]; Makhachkala, Kochubei [Ponomarev et al., 2011b]; Sarykum sand dune [Ponomarev, Abdurakhmanov, 2014].

MATERIAL. Russia, Dagestan: 1 \%, [34], 1.10.2012 (G.Kh.); $1 \mathrm{O}^{7}$, [19], 05.2013 (G.Kh.).

\section{SPARASSIDAE}

Micrommata virescens (Clerck, 1758)

RECORDS. Russia, Dagestan: Madzhalis [Ponomarev, Alieva, 2010; Abdurakhmanov, Alieva, 2011]; Bryanskaya Kosa, Tyuleniy Island [Ponomarev et al., 2011a]; Babayurt [Ponomarev et al., 2011b]. Ma)

MATERIAL. Russia, Dagestan: 1 +, [2], 23.06.2012 (Rabadi-

\section{TETRAGNATHIDAE}

Metellina segmentata (Clerck, 1758)

RECORDS. Russia, Dagestan: Verkhny Karanay [Ponomarev, Khalidov, 2007; Khalidov, Ponomarev, 2008]; Kondik, Maydanskoe, Inkhokvari, Khonokh, Khvarshi [Abdura- 
khmanov, Alieva, 2009, 2011]; Dakhadaevka, Sulak [Ponomarev et al., 2011b] G.Kh.)

MATERIAL. Russia, Dagestan: $1 \overbrace{}^{\top}, 10$ 우, [9], 4.09.2013

Pachygnatha degeeri Sundevall, 1830

RECORDS. Russia, Dagestan: Erpeli, Verkhny Karanay [Ponomarev, Khalidov, 2007; Khalidov, Ponomarev, 2008]; Tsekhok [Ponomarev et al., 2008, 2011b]; Irganay, Kondik [Abdurakhmanov, Alieva, 2009, 2011]; Amsar [Abdurakhmanov et al., 2012]. mova)

MATERIAL. Russia, Dagestan: 1 \% [32], 27.10 .2012 (Gali-

Tetragnatha dearmata Thorell, 1873

RECORDS. Russia, Dagestan: Nizhnee Kazanishche [Ponomarev et al., 2011b]; Sarykum sand dune [Ponomarev et al., 2017b]. (E.I.).

MATERIAL. Russia, Dagestan: 1 +, [24], beach, 5.06.2013

Tetragnatha extensa (Linnaeus, 1758)

RECORDS. Russia, Dagestan: Kondik [Abdurakhmanov, Alieva, 2009, 2011]; Bryanskaya Kosa [Ponomarev et al., 2011a].

MATERIAL. Russia, Dagestan: $2 \sigma^{7} \sigma^{7}, 2$ 우, [9], nr. lake, 15.07.2017 (M.A.).

Tetragnatha montana Simon, 1874

RECORDS. Russia, Dagestan: Kondik, Samur forest [Abdurakhmanov, Alieva, 2009, 2011]; Bogatyrevka, Nizhnee Kazanishche [Ponomarev et al., 2011b]; Gasha, Makhachkala [Abdurakhmanov, Alieva, 2011].

MATERIAL. Russia, Dagestan: $1 \sigma^{7}, 2$ 우, [2], 23.06.2011 (G.Kh.); 1 ○', 1 +, [23], 16-18.06.2014 (G.Kh.).

Tetragnatha nigrita Lendl, 1886

RECORDS. Russia, DAgESTAN: Samur forest [Abdurakhmanov, Alieva, 2009, 2011].

MATERIAL. RusSia, Dagestan: $19,[19]$, at the foot of Sarykum sand dune, 06.2009 (G.Kh.); 1 O', [2], 23.06.2011 (G.Kh.).

Tetragnatha obtusa C.L. Koch, 1837

RECORDS. Russia, Dagestan: Kurush [Abdurakhmanov, Alieva, 2009, 2011]; Gasha [Abdurakhmanov, Alieva, 2011]; Bryanskaya Kosa [Ponomarev et al., 2011a]; Sarykum sand dune [Ponomarev et al., 2017b].

MATERIAL. Russia, Dagestan: $10^{7}$, [2], 23.06.2011 (G.Kh.).

\section{THERIDIIDAE}

Asagena meridionalis Kulczyński in Chyzer et Kulczyński, 1894

RECORDS. Russia, Dagestan: Gasha, Madzhalis [Ponomarev, Alieva, 2010: Steatoda; Abdurakhmanov, Alieva, 2011: Steatoda]; Sarykum sand dune [Ponomarev et al., 2017b].

MATERIAL. Russia, Dagestan: $1+$, [13], forest, 17.07.2013 (Galimova).

\section{Asagena phalerata (Panzer, 1801)}

RECORDS. Russia, Dagestan: Dzhaba, Khunzakh [Ponomarev et al., 2008b; Steatoda]; Kurush, Makhachkala, Kondik, Chuvek, Tsuduk [Abdurakhmanov, Alieva, 2009, 2011: in both cases Steatoda]; Bryanskaya Kosa, Tyuleniy Island [Ponomarev et al., 2011a]; Ingishi, Inchkhe, Yuzhno-
Sukhokumsk [Abdurakhmanov et al., 2012]; Sarykum sand dune, Chechen Island [Ponomarev, Abdurakhmanov, 2014]; Sarykum sand dune [Ponomarev et al., 2017b].

MATERIAL. Russia, Dagestan: 2 +\%, [29], 20.07.2017 (M.A.).

Enoplognatha latimana Hippa et Oksala, 1982

RECORDS. Russia, Dagestan: Shalbuzdag Mt. [Ponomarev et al., 2008]; Irganay, Kurush, Kondik, Chuvek, Tsuduk [Abdurakhmanov, Alieva, 2009, 2011]; Gasha [Abdurakhmanov, Alieva, 2011]; Nizhnee Kazanishche [Ponomarev et al., 2011b]; Ingishi [Abdurakhmanov et al., 2012].

MATERIAL. Russia, Dagestan: $1 \sigma^{7}$, [9], 6.07.2011 (G.Kh.);

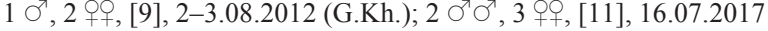
(M.A.); 1 +, [29], 20.07.2017 (M.A.).

\section{Enoplognatha oelandica (Thorell, 1875)}

RECORDS. Russia, Dagestan: Verkhnee Kazanishche [Ponomarev et al., 2008]; Archib, Kurush [Abdurakhmanov, Alieva, 2009, 2011]; Sarykum sand dune [Ponomarev et al., 2017b].

MATERIAL. Russia, Dagestan: 1 +, [11], 16.07.2017 (M.A.); 1 \%, [29], 20.07.2017 (M.A.); $4 \bigcirc^{7} \sigma^{7}$, [19], tidal saltmarsh, 14.045.05.2018 (M.A.).

Enoplognatha thoracica (Hahn, 1833)

RECORDS. Russia, DAGESTAN: Sarykum sand dune [Ponomarev, Abdurakhmanov, 2014; Ponomarev et al., 2017b].

MATERIAL. Russia, Dagestan: 1 +, [9], 6.07.2011 (G.Kh.); 1 †, [19], 14.05.2014 (G.Kh.).

Episinus truncatus Latreille, 1809

RECORDS. Russia, Dagestan: Gasha, Makhachkala [Ponomarev, Alieva, 2010; Abdurakhmanov, Alieva, 2011]; Sarykum sand dune [Ponomarev, Abdurakhmanov, 2014].

MATERIAL. Russia, Dagestan: 2 우, [9], 2-3.08.2012 (G.Kh.).

Kochiura aulica (C.L. Koch, 1828)

RECORDS. Russia, Dagestan: Bryanskaya Kosa, Tyuleniy Island [Ponomarev et al., 2011a]; Yuzhno-Sukhokumsk [Abdurakhmanov et al., 2012]; Chechen Island [Ponomarev, Abdurakhmanov , 2014].

MATERIAL. Russia, Dagestan: 1 \%, [24], beach, 5.06.2013 (E.I.); 1 +, [8], 28.05.2017 (M.A.); 1 \%, [30], nr. pond, 28.05.2017 (M.A.); 1 O , 1 \%, [27], 12.06.2017 (M.A.).

Latrodectus tredecimguttatus (P. Rossi, 1790)

RECORDS. Russia, Dagestan: Terek-Nogay steppes, Tabasaransky Distr., Akhty [Rossikov, 1904]; Kizlyar [Moritz, 1914]; Chechen Island [Ponomarev, Khalidov, 2007; Ponomarev et al., 2011a; Ponomarev, Abdurakhmanov, 2014]; Aimaki [Ponomarev et al., 2008]; Tyuleniy Island [Ponomarev et al., 2011a]; Nizhnee Kazanishche [Ponomarev et al., 2011b]; Yuzhno-Sukhokumsk [Abdurakhmanov et al., 2012]; Sarykum sand dune [Ponomarev et al., 2017b].

MATERIAL. Russia, Dagestan: 1 juv., [18], tidal saltmarsh, 17.06.2017 (M.A.)

Parasteatoda tepidariorum (C.L. Koch, 1841)

RECORDS. Russia, Dagestan: Erpeli, Verkhnee Kazanishche [Ponomarev, Khalidov, 2007: Achaearanea; Khalidov, Ponomarev, 2008: Achaearanea]; Verkhnee Kazanishche, Nizhnee Kazanishche, Dzhengutai [Ponomarev et al., 2008: Achaearanea]; Verkhny Gunib [Ponomarev et al., 2011b]; Gasha, Makhachkala [Abdurakhmanov, Alieva, 2011].

MATERIAL. Russia, Dagestan: 1 +, [23], 16-18.06.2014 (G.Kh.). 

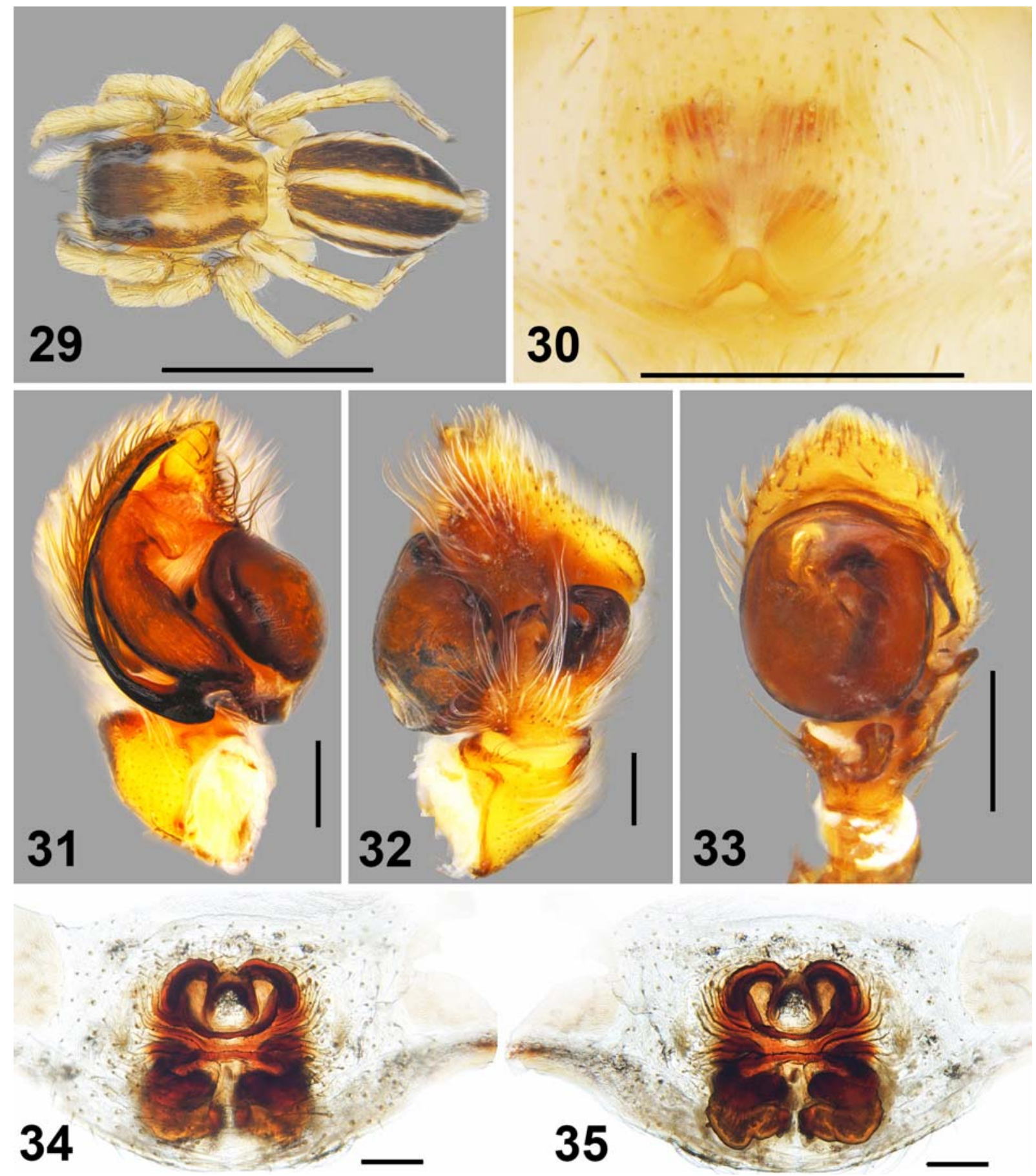

Figs 29-35. Habitus and copulatory organs of Phlegra lineata (C.L. Koch, 1846) (29, 30), Yllenus zyuzini Logunov et Marusik, 2003 (31, 32), Xysticus kaznakovi Utochkin, 1968 (33) and X. loeffleri Roewer, 1955 (34, 35): 29 - female, dorsal view; 30, 34 - epigyne, ventral view; 35 - spermathecae, dorsal view; $31-33$ - male palp (31 - ventro-prolateral view; 32 - retrolateral view; 33 - ventral view). Scale bars: (29) $2.5 \mathrm{~mm},(30) 0.5 \mathrm{~mm},(31-35) 0.25 \mathrm{~mm}$.

Рис. 29-35. Габитус и копулятивные органы Phlegra lineata (C.L. Koch, 1846) (29, 30), Yllenus zyuzini Logunov et Marusik, 2003 (31, 32), Xysticus kaznakovi Utochkin, 1968 (33) и X. loeffleri Roewer, 1955 (34, 35): 29 — самка, вид сверху; 30, 34 — эпигина, вид снизу; 35 - сперамтека, вид сверху; 31-33 - пальпа самца (31 - вентро-пролатерально; 32 - ретролатерально; 33 вентрально). Масштаб: (29) 2,5 мм, (30) 0,5 мм, (31-35) 0,25 мм. 
Phylloneta impressa (L. Koch, 1881)

RECORDS. Russia, Dagestan: Kondik, Chuvek, Tsuduk [Abdurakhmanov, Alieva, 2009, 2011: Theridion impressum]; Gasha, Bolshie Turali [Abdurakhmanov, Alieva, 2011]; Kumukh [Ponomarev et al., 2011b]; Yuzhno-Sukhokumsk [Abdurakhmanov et al., 2012]; Sarykum sand dune [Ponomarev et al., 2017b].

MATERIAL. Russia, Dagestan: 1 +, [9], 6.07 .2011 (G.Kh.); 1 $\sigma^{7}, 1$ ㅇ, [9], 27.07-2.08.2012 (G.Kh.); $1 \bigcirc^{7}$, [37], field, 11.06.2013 (G.Kh.); 2 O' O' $^{7}$ [8], 28.05 .2017 (M.A.); 1 O', [27], 12.06.2017 (M.A.); $1 \sigma^{7},[11], 16.07 .2017$ (M.A.).

\section{Platnickina tincta (Walckenaer, 1802)}

RECORDS. Russia, Dagestan: Aglobi [Abdurakhmanov, Alieva, 2009, 2011]; Gasha [Abdurakhmanov et al., 2012].

MATERIAL. Russia, Dagestan: 1 +, [9], 6.07.2011 (G.Kh.); 1 ㅇ, [9], 30.05.2018 (M.A.).

Steatoda albomaculata (De Geer, 1778)

RECORDS. Russia, Dagestan: Dzhaba [Ponomarev et al., 2008]; Kurush, Maydanskoe, Kondik [Abdurakhmanov, Alieva, 2009, 2011]; Bryanskaya Kosa, Tyuleniy Island, Chechen Island [Ponomarev et al., 2011a]; Yuzhno-Sukhokumsk [Abdurakhmanov et al., 2012]; Sarykum sand dune, Chechen Island [Ponomarev, Abdurakhmanov, 2014]; Sarykum sand dune [Ponomarev et al., 2017b].

MATERIAL. Russia, Dagestan: 2 +9, [19], 05.2013 (G.Kh.); 3 우, [11], 16.07.2017 (M.A.); 16 우, [29], 20.07.2017 (M.A.).

\section{Steatoda castanea (Clerck, 1758)}

RECORDS. Russia, Dagestan: Verkhnee Kazanishche, Erpeli [Ponomarev, Khalidov, 2007; Khalidov, Ponomarev, 2008]; Aimaki, Buynaksk, Verkhnee Kazanishche, Nizhnee Kazanishche, Verkhny Gunib, Dzhaba, Dubki, Tsekhok, Khunzakh Distr. [Ponomarev et al., 2008b]; Gasha [Abdurakhmanov, Alieva, 2011]; Buynaksk, Nizhnee Kazanishche, Verkhny Gunib, Makhachkala [Ponomarev et al., 2011b]; Inchkhe [Abdurakhmanov et al., 2012].

MATERIAL. Russia, Dagestan: 1 , [13], 5.08.2012, (Galimova).

Steatoda dahli (Nosek, 1905)

RECORDS. Russia, Dagestan: Bryanskaya Kosa [Ponomarev et al., 2011a]; Chechen Island [Ponomarev, Abdurakhmanov, 2014].

MATERIAL. Russia, Dagestan: $1 \sigma^{7}$, [21], sagebrush, 16.06 . 2013 (E.I.); 1 Ơ $^{7} 1$ +, [18], tidal saltmarsh, 17.06.2017 (M.A.).

Steatoda grossa (C.L. Koch, 1838)

RECORDS. Russia, Dagestan: Verkhny Dzhengutai [Ponomarev et al., 2008]; Makhachkala [Abdurakhmanov, Alieva, 2011]; Nizhnee Kazanishche [Ponomarev et al., 2011b].

MATERIAL. Russia, Dagestan: $10^{7}$, [33], Leninsky Komsomol Park, 28.10.2012 (G.Kh.).

Steatoda paykulliana (Walckenaer, 1806)

RECORDS. Russia, Dagestan: Erpeli [Ponomarev, Khalidov, 2007; Khalidov, Ponomarev, 2008]; Buynaksk, Verkhnee Kazanishche, Karabudakhkent [Ponomarev et al., 2008]; Kurush, Kondik, Chuvek, Tsuduk [Abdurakhmanov, Alieva, 2009, 2011]; Chechen Island [Ponomarev et al., 2011a]; Novy Kumukh, Novokuli, Sulak [Ponomarev et al., 2011b]; Aglobi, Gasha, Turali, Tselyagyun [Abdurakhmanov, Alieva, 2011]; Bryansk, Inchkhe [Abdurakhmanov et al., 2012]; Sarykum sand dune [Ponomarev, Abdurakhmanov, 2014; Ponomarev et al., 2017b].
MATERIAL. Russia, Dagestan: 1 \%, [19], 20.06 .2009 (G.Kh.); $1 \mathrm{O}^{\top}, 1$ +, [19], 9.03 .2013 (G.Kh.); 1 +, [19], 05.2013 (G.Kh.); 1 , [31], 30.04.2017 (M.A.)

Steatoda triangulosa (Walckenaer, 1802)

RECORDS. Russia, Dagestan: Aimaki, Buynaksk, Verkhnee Kazanishche, Dubki, Nizhnee Kazanishche [Ponomarev et al., 2008]; Aglobi, Makhachkala, Tselyagyun [Abdurakhmanov, Alieva, 2011]; Nizhnee Kazanishche [Ponomarev et al., 2011b] (G.Kh.).

MATERIAL. Russia, Dagestan: $1 \sigma^{\Uparrow}, 2$ 우, [33], 2.09.2012

Theridion cinereum Thorell, 1875

material. Russia, Dagestan: $1 \sigma^{7}, 3$ of , [33], $12-$ 26.06.2011 (G.Kh.); 1 , [33], 20.07.2012 (G.Kh.).

COMMENTS. The new record for the fauna of Dagestan. The species in known from central and southern Europe, Azerbaijan, Turkey, Central Asia [WSC, 2018]; it was also recorded from the NW Caucasus [Spassky, 1937; Ponomarev, Volkova, 2013].

Theridion pictum (Walckenaer, 1802)

MATERIAL. Russia, Dagestan: 1 \%, [9], 3.08 .2012 (G.Kh.).

COMMENTS. The new record for the fauna of Dagestan. In the Caucasus, it was reported from Azerbaijan [Otto, 2017]. The record of this species from ChechenoIngushetia (now Chechnya and Ingushetia republics, Russia) [Minoransky et al., 1984] is erroneous and actually belongs to Theridion hemerobium Simon, 1914.

\section{THOMISIDAE}

Cozyptila guseinovorum Marusik et Kovblyuk, 2005 MATERIAL. Russia, Dagestan: $10^{7}$, [23], 5.06.2013 (E.I.).

COMMENTS. The new record for the fauna of Dagestan. This species is widespread across the Caucasus [Otto, 2017].

Ebrechtella tricuspidata (Fabricius, 1775)

RECORDS. Russia, DAGESTAN: Erpeli, Verkhny Karanay [Ponomarev, Khalidov, 2007; Khalidov, Ponomarev, 2008]; Buynaksk [Ponomarev et al., 2008]; Makhachkala, Maydanskoe [Abdurakhmanov, Alieva, 2009, 2011]; Nizhnee Kazanishche, Shamkhal [Ponomarev et al., 2011b]; Sulak [Ponomarev, Abdurakhmanov, 2014].

MATERIAL. Russia, Dagestan: 1 9, [9], 6.08 .2012 (G.Kh.); 1 + , [5], meadow, $730 \mathrm{~m}$ a.s.1., 19.06.2017 (M.A.).

Heriaeus graminicola (Doleschall, 1852)

MATERIAL. Russia, Dagestan: 1 \%, [37], 06.2012 (D. Magomedova); 1 o $^{7}, 2$ 우, [23], 25.06 .2017 (M.A.).

COMMENTS. The new record for the fauna of Dagestan. This species is knonw from southern and central Europe, and Central Asia [WSC, 2018].

Heriaeus oblongus Simon, 1918

RECORDS. Russia, DAgEstan: Bryanskaya Kosa [Ponomarev et al., 2011a: Heriaeus melloteei]; Erpeli [Ponomarev et al., 2011b: Heriaeus melloteei]; Yuzhno-Sukhokumsk [Abdurakhmanov et al., 2012].

MATERIAL. Russia, Dagestan: 1 9, [25], 28.05 .2017 (M.A.); 2 우, [8], 28.05.2017 (M.A.); 1 +, [18], 4.06 .2017 (M.A.); 1 O', 1 +, [35], 6.06.2013 (G.Kh.); 1 O', 1 +, [36], 6.06.2013 (G.Kh.).

Misumena vatia (Clerck, 1758)

RECORDS. Russia, DAGESTAN: Verkhny Karanay [Ponomarev, Khalidov, 2007; Khalidov, Ponomarev, 2008]; May- 
danskoe [Abdurakhmanov, Alieva, 2009, 2011]; Gasha, Tselyagyun [Abdurakhmanov, Alieva, 2011]; Tsekhok [Ponomarev et al., 2011b].

MATERIAL. Russia, Dagestan: 2 우, [9], 3.08 .2012 (G.Kh.); $1 \mathrm{O}^{\mathrm{T}}$, [27], 12.06.2017 (M.A.).

Ozyptila lugubris (Kronederg, 1875)

RECORDS. Russia, Dagestan: Chechen Island [Ponomarev, Khalidov, 2007]; Bryanskaya Kosa [Ponomarev et al., 2011a].

MATERIAL. Russia, Dagestan: 1 O', [6], 9.04.2017 (E.I.).

Runcinia grammica (C.L. Koch, 1837)

RECORDS. Russia, DAgestan: Maydanskoe [Abdurakhmanov, Alieva, 2009, 2011]; Gasha [Abdurakhmanov, Alieva, 2011]; Bryanskaya Kosa, Tyuleniy Island [Ponomarev et al., 2011a]; Bogatyrevka [Ponomarev et al., 2011b]; Yuzhno-Sukhokumsk [Abdurakhmanov et al., 2012]; Sarykum sand dune [Ponomarev et al., 2017b].

MATERIAL. Russia, Dagestan: $1 \sigma^{7}$, [36], 6.06 .2013 (G.Kh.); $1 \mathrm{O}^{7}$, [37], field, 11.06.2013 (G.Kh.); $7 \mathrm{O}^{\top} \mathrm{O}^{7}, 8$ + + , [27], 12.06.2017 (M.A.); $2 \sigma^{7} \sigma^{\top}, 1$ +, [23], 25.06 .2017 (M.A.).

Synema globosum (Fabricius, 1775)

RECORDS. Russia, Dagestan: Erpeli [Ponomarev, Khalidov, 2007; Khalidov, Ponomarev, 2008]; Verkhny Dzhengutai [Ponomarev et al., 2008]; Maydanskoe, Kondik, Chuvek, Tsuduk [Abdurakhmanov, Alieva, 2009, 2011]; Gasha [Abdurakhmanov, Alieva, 2011]; Novyi Kumukh [Ponomarev et al., 2011b].

MATERIAL. Russia, Dagestan: $1 \sigma^{7}$, [9], 6.07 .2011 (G.Kh.); 1 ㅇ, [9], 6.08.2012, (G.Kh.); 1 ㅇ, [5], meadow, $730 \mathrm{~m}$ a.s.l., 19.06.2017 (M.A.); 1 O', 2 \%क, [23], 25.06.2017 (M.A.).

Thomisus onustus Walckenaer, 1805

RECORDS. Russia, Dagestan: Nizhnee Kazanishche, Verkhnee Kazanishche, Nizhny Dzhengutai, Karabudakhkent [Ponomarev et al., 2008]; Maydanskoe, Kondik, Chuvek, Tsuduk [Abdurakhmanov, Alieva, 2009, 2011]; Gasha [Abdurakhmanov, Alieva, 2011]; Bryanskaya Kosa, Chechen Island [Ponomarev et al., 2011a]; Nizhnee Kazanishche, Novyi Kumukh [Ponomarev et al., 2011b]; Yuzhno-Sukhokumsk [Abdurakhmanov et al., 2012]; Chechen Island [Ponomarev, Abdurakhmanov, 2014]; Sarykum sand dune [Ponomarev et al., 2017b].

MATERIAL. Russia, DAgestan: 1 + , [19], 06.2009 (S. Gamzatova); 1 , [19], 2.07.2013 (P.I. Tagirova, M.M. Shakhmanova); 1 +, [9], 2.08.2012 (G.Kh.); $2 \sigma^{7} \sigma^{7}$, [35], 6.06.2013 (G.Kh.); $8 \sigma^{3} \sigma^{7}$, [36], 6.06.2013 (G.Kh.); $1 \mathrm{O}^{7}$, [3], 06.2013 (Abdullaeva, Magomedova); 1 +, [25], 28.05 .2017 (M.A.); 1 O$^{\top}$, [8], 28.05 .2017 (M.A.); 3 O $^{7}, 1$ +, [27], 12.06 .2017 (M.A.); 2 우, [29], 20.07.2017 (M.A.).

Tmarus punctatissimus (Simon, 1870)

MATERIAL. Russia, DAGESTAN: $1 \sigma^{7}$, [13], forest, 28.06.2013 (Galimova); $1 \sigma^{7}$, [23], 16-18.06.2014 (G.Kh.).

COMMENTS. The new record for the fauna of Dagestan. In the Caucasus, it was also recorded from South Ossetia [Ponomarev, Komarov, 2015].

Xysticus acerbus Thorell, 1872

RECORDS. Russia, Dagestan: Buynaksk [Ponomarev et al., 2008]; Tselyagyun [Abdurakhmanov, Alieva, 2011]; Sarykum sand dune [Ponomarev et al., 2017b].

MATERIAL. Russia, Dagestan: 1 o , [19], 04-05.2014 (G.Kh.); 1 \%, [5], meadow, $730 \mathrm{~m}$ a.s.1., 19.06.2017 (M.A.).

Xysticus bifasciatus C.L. Koch, 1837

RECORDS. Russia, Dagestan: Kondik [Abdurakhmanov, Alieva, 2009, 2011].
MATERIAL. Russia, Dagestan: $1 \sigma^{7}$, [12], 15.07.2017 (M.A.).

Xysticus cristatus (Clerck, 1758)

RECORDS. Russia, Dagestan: Verkhny Gunib, Khunzakh [Ponomarev et al., 2008]; Tsekhok [Ponomarev et al., 2008, 2011b]; Kurush [Abdurakhmanov, Alieva, 2009]; Chechen Island [Ponomarev, Abdurakhmanov, 2014].

MATERIAL. Russia, Dagestan: 1 + , [9], 6.08.2012 (G.Kh.); 1 ㅇ, [35], 6.06.3013 (G.Kh.); 1 + , [26], 11.06.2017 (G.Kh.).

\section{Xysticus kaznakovi Utochkin, 1968} Fig. 33.

Xysticus kaznakovi Utochkin, 1968: 39, figs 118-120 ( $\left.\sigma^{7}\right)$. Xysticus kaznakovi: Marusik, Logunov, 1990: 42, figs 27-28 $\left(\sigma^{7}\right)$

Xysticus kaznakovi: Kiany et al., 2017: 6, figs 11a-e ( $\sigma^{7}+$ )

MATERIAL. Russia, Dagestan: $1 \sigma^{7}$, [21], ravine slope with sagebrush, 12.05.2013 (E.I.).

COMMENTS. The new record for the fauna of Russia. This species was known from the eastern Mediterranean, Iran, Azerbaijan, Turkmenistan, and Tajikistan [Mikhailov, 2013; Kiany et al., 2017; Nentwig et al., 2018]. The valley of Shura-Ozen' in Dagestan is the northernmost locality for this species. The palp conformation of the male studied (Fig. 33 ) is in agreement with earlier published illsutrations [Marusik, Logunov, 1990; Kiany et al., 2017].

Xysticus kochi Thorell, 1872

RECORDS. Russia, Dagestan: Verkhnee Kazanishche, Verkhny Karanay [Ponomarev, Khalidov, 2007; Khalidov, Ponomarev, 2008]; Buynaksk, Khuchni [Ponomarev et al., 2008]; Aglobi, Maydanskoe [Abdurakhmanov, Alieva, 2009, 2011]; Gasha, Madzhalis, Turali, Tselyagyun [Abdurakhmanov, Alieva, 2011]; Bryanskaya Kosa [Ponomarev et al., 2011a]; Sarykum sand dune, Nizhnee Kazanishche, Novyi Kumukh, Kisilyurt, Sultan-Yangiyurt, Talgi Canyon [Ponomarev et al., 2011b]; Inchkhe, Ullubievka, Novokuli, Tarumovka [Abdurakhmanov et al., 2012]; Sarykum sand dune [Ponomarev, Abdurakhmanov, 2014; Ponomarev et al., 2017b].

MATERIAL. Russia, Dagestan: 1 \%, [33], 3.06 .2009 (N.M. Gasanova); 1 ㅇ, [2], 23.06.2011 (G.Kh.); 1 \%, [6], 15.04.2012 (G.Kh.); 1 \%, [19], 16.04.2012 (G.Kh.); 1 \%, [19], forest stand, 9.06.2013 (G.Kh.); 3 O $^{7}, 1$ ㅇ, [21], 20.04.2013 (E.I.); 4 O $^{7} \sigma^{7}$, [21], sagebrush, 5.05.2013 (E.I.); $3 O^{7} \sigma^{7}, 1+$, [21], ravine slope with sagebrush, 12.05.2013 (E.I.); 1 +, [21], 25.05.2013 (E.I.); 11 $\sigma^{7} \sigma^{7},[21], 16.06 .2013$ (E.I.); 1 ㅇ, [21], sagebrush, 16.06.2013 (E.I.); 2 Oᄋ , [35], 6.06.2013 (G.Kh.); 1 O', [36], 6.06.2013 (G.Kh.) 1 O', [23], 16-18.06.2014 (G.Kh.); 1 우, [23], 25.06.2017 (M.A.); 1 + , [8], 28.05.2017 (M.A.); 1 +, [18], 10.06.2017 (M.A.); 1 + , [18], tidal saltmarsh, 17.06.2017 (M.A.); 1 +, [27], 12.06.2017 (M.A.).

Xysticus laetus Thorell, 1875

RECORDS. Russia, Dagestan: Bolshie Turali [Ponomarev, Alieva, 2010; Abdurakhmanov, Alieva, 2011]; Bryanskaya Kosa, Tyuleniy Island [Ponomarev et al., 2011a]; Sarykum sand dune [Ponomarev et al., 2011b, 2017b].

MATERIAL. Russia, Dagestan: 1 +, [2], 14.06.2013 (G.Kh.).

Xysticus loeffleri Roewer, 1955

Figs 34, 35.

Xysticus löffleri Roewer, 1955: 777, fig. $25(+)$.

Xysticus turanicus: Marusik, Logunov, 1990: 45, figs 39-41 $\left(O^{\top}+\right)$.

Xysticus loeffleri: Kiany et al., 2017: 6, figs 12f-b ().

MATERIAL. Russia, Dagestan: 1 9, [6], 15.04.2012 (G.Kh.); 2 우, [15], 19.04.2014 (E.I.); 1 ㅇ, [31], 30.04.2017 (M.A.). 
COMMENTS. The new record for the fauna of Russia. This species was known from Greece, Turkey, Iran and Central Asia [WSC, 2018]. The localities in Dagestan are the northernmost records for this species; this is why illustrations of the specimens studied (Figs 34, 35) are provided.

Xysticus luctator L. Koch, 1870

RECORDS. Russia, Dagestan: Buynaksk Distr. [Ponomarev et al., 2008]. (E.I.).

MATERIAL. Russia, Dagestan: $3 \sigma^{7} \sigma^{7}$, [23], 5-26.06.2013

Xysticus tristrami (O. Pickard-Cambridge, 1872)

RECORDS. Russia, DAgESTAN: Makhachkala [Ponomarev et al., 2008]; Bryanskaya Kosa, Tyuleniy Island, Chechen Island [Ponomarev et al., 2011a]; Sarykum sand dune, Chechen Island [Ponomarev, Abdurakhmanov, 2014]; Sarykum sand dune [Ponomarev et al., 2017b].

MATERIAl. Russia, Dagestan: $7 \bigcirc^{7} \bigcirc^{7}, 2$ q, [18], 10 17.06.2017 (M.A.); 1 ㅇ, [4], nr. water-power plant, 8.05.2017 (M.A.).

\section{TITANOECIDAE}

Nurscia albomaculata (Lucas, 1846)

RECORDS. Russia, Dagestan: Nordovyi Island [Ponomarev et al., 2011a].

MATERIAL. Russia, Dagestan: $10^{7}$, [3], 06.2011 (Gasanov); $1 \sigma^{\top},[3], 11.06 .2013$ (Alibekova, Abdusalimova).

Nurscia albosignata Simon, 1874

RECORDS. Russia, Dagestan: Tselyagyun [Ponomarev, Alieva, 2010; Abdurakhmanov, Alieva, 2011].

MATERIAL. Russia, Dagestan: 1 \%, [33], 20.07.2012 (G.Kh.).

Titanoeca turkmenia Wunderlich, 1995

RECORDS. Russia, Dagestan: Bryanskaya Kosa, Nordovyi Island [Ponomarev et al., 2011a]; Yuzhno-Sukhokumsk [Abdurakhmanov et al., 2012].

MATERIAL. Russia, Dagestan: $1 \sigma^{7}, 1$ o, [2], 23.06.2012 (Rabadinova); $1 \mathrm{O}^{7}$, [18], 10.06.201 (M.A.).

\section{ULOBORIDAE}

Uloborus walckenaerius Latreille, 1806

RECORDS. Russia, DAgESTAN: Bryanskaya Kosa [Ponomarev et al., 2011a]; Yuzhno-Sukhokumsk [Abdurakhmanov et al., 2012].

MATERIAL. Russia, Dagestan: 1 + , [9], 2.09 .2012 (G.Kh.); 1 +, [33], 2.09.2012 (G.Kh.).

\section{ZODARIIDAE}

Zodarion morosum Denis, 1935

RECORDS. Russia, Dagestan: Tarki-Tau Mt. [Ponomarev et al., 2008]; Turali, Gasha [Abdurakhmanov, Alieva, 2011]; Bryanskaya Kosa, Tyuleniy Island, Chechen Island [Ponomarev et al., 2011a]; Sarykum sand dune, Chechen Island [Ponomarev, Abdurakhmanov, 2014]; Sarykum sand dune [Ponomarev et al., 2017b].

MATERIAL. Russia, Dagestan: $30^{\top} \sigma^{\top}, 2 \circ 9$, [21], sagebrush, 5.05-16.06.2013 (E.I.); 1 \%, [21], ravine slope with sagebrush, 12.05.2013 (E.I.); $11 \mathrm{O}^{7} \sigma^{7}, 1$ ㅇ, [21], 25.05.2013 (E.I.); $1 \mathrm{O}^{7}, 3$ 우, [19], 05.2013 (G.Kh.); 1 O , [36], 6.06.2013 (G.Kh.); 1 ․, [14], gorge, under stones, 18.06.2017 (M.A.).

\section{Discussion}

The list presented above contains 223 spider species in 28 families. Fourteen species (Dictyna armata, D. ottoi, Berlandina saraevi, Nomisia conigera, Nomisia ripariensis, Talanites dunini, Pholcus sogdianae, Aelurillus concolor, Chalcoscirtus tanasevichi, Chinattus caucasicus, Phlegra lineata, Yllenus zyuzini, Xysticus kaznakovi, X. loeffleri) have been recorded from Russia for the first time. In addition, 31 species (Pireneitega ovtchinnikovi, Amaurobius erberi, Brigittea latens, Nigma flavescens, Harpactea spasskyi, Cheiracanthium gratum, Callilepis nocturna, Drassyllus crimeaensis, Kishidaia conspicua, Micaria sociabilis, Trachyzelotes jaxartensis, T. manytchensis, Zelotes khostensis, Agyneta saaristoi, Gongylidium rufipes, Oedothorax apicatus, Alopecosa pentheri, Philodromus poecillus, Tibellus maritimus, Phrurolithus pullatus, Aelurillus lutosus, Calositticus caricis, C. inexpectus, Euophrys frontalis, Pellenes geniculatus, Salticus zebraneus, Theridion cinereum, T. pictum, Cozyptila guseinovorum, Heriaeus graminicola, Tmarus punctatissimus) are new records for Dagestan. Two species (Talanites cf. dunini, Lycosa cf. praegrandis) remains undetermined. Thus, currently the entire spider fauna of Dagestan consists of no less than 460 species in 32 families, including the results of this paper.

In the course of the present study, a special attention was paid to the araneofauna of the least studied northern lowlands of Dagenstan. With the available literature data [Abdurakhmanov et al., 2012; Ponomarev, Abdurakhmanov, 2014; Ponomarev et al., 2017b], the species number of northern Dagestan has reached 240 , which is significantly higher than that of neighbouring Kalmykia with only 180 species recorded [Minoransky, Ponomarev, 1984]. The spider species list of the Sarykum sand dune site of the Dagestansky Reserve has been notably extended and now contains 160 species. The territory of Dagestan seems to be the best studied administrative unit of the Russian Caucasus. For instance, only 180 spider species have been recorded from Chechnya and Ingushetia [Minoransky et al., 1984; Minoransky, 1988], 252 species from Adygea [Ponomarev et al., 2012], some 360 species from North Ossetia [Ponomarev, Komarov, 2013], and 265 from Starvropol Territory [Ponomarev et al., 2017a]. However, compared to the spider faunas of Azerbaijan and Georgia, with 619 and 716 species recorded accordingly [Otto, 2017], the fauna of Dagestan looks rather depauperate. In our opinion, a much higher spider diversity can be expected in Dagestan, and thus further taxonomic-faunistic research is to continue.

Acknowledgments. The authors are much obliged to Elena Il'ina (The Caspian Institute of Biological Resources of the Russian Academy of Sciences, «Dagestansky» Reserve) for the material provided for the present study and its initial sorting, and to Maxim Nabozhenko (the same institution) for his help with English translation of the text. The 
work was supported by the government contract for the Southern Scientific Centre of RAS, project AAAA-A19119011190176-7 for Alexander Ponomarev and Vladimir Shmanko.

\section{References}

Abdurakhmanov G.M., Alieva S.V. 2009. [Composition and Geographical Distribution of the Spiders (Aranei) in the Republic Dagestan] // Yugh Rossii: ecologiya, razvitie. No.3. P.38-50 [in Russian, with English summary].

Abdurakhmanov G.M., Alieva S.V. 2011. [Results of a study on the spider fauna (Aranei) of Dagestan] // Yugh Rossii: ecologiya, razvitie. No.1. P.44-66 [in Russian, with English summary].

Abdurakhmanov G.M., Ponomarev A.V., Alieva S.V. 2012. [Spiders (Arachnida: Aranei) of the Republic Dagestan: Species Composition, Distribution]. Makhachkala: Dagestan State Pedagogic University. 220 p. [In Russian]

Azarkina G.M. 2003. Aelurillus ater (Kroneberg, 1875) and related species of jumping spiders in the fauna of Middle Asia and the Caucasus (Aranei: Salticidae) // Arthropoda Selecta. Vol.11 (for 2002). No.1. P.89-107.

Azarkina G.M., Mirshamsi O. 2014. Description of a new Aelurillus species from Khorasan province of Iran, with comments on A. concolor Kulczyñski 1901 (Araneae: Salticidae) // Zoology in the Middle East. Vol.60. No.1. P.82-91.

Bosmans R., Blick T. 2000. Contribution to the knowledge of the genus Micaria in the West-palaearctic region, with description of the new genus Arboricaria and three new species // Mem. Soc. entomol. ital. Vol.78. Fasc.2. P.443-476.

Brignoli P.M. 1978. Ragni di Turchia V. Specie nuove o interessanti, cavernicole ed epigee, di varie famiglie (Araneae) // Revue Suisse de Zoologie. T.85. Fasc.3. P.461-541.

Buchar J., Thaler K. 1998. Lycosidae from the high alpine zone of the Caucasus range, with comparative remarks on the fauna of the Alps (Arachnida: Araneae) // Linzer Biologische Beiträge. Bd.30. H.2. P.705-717.

Chatzaki M. 2010. A revision of the genus Nomisia in Greece and neighboring regions with the description of two new species // Zootaxa. Vol.2501. P.1-22.

Dunin P.M. 1984. [Fauna and ecology of the spiders of the Apscheron Peninsula, Azerbadjan SSR] // Fauna I ekologiua paukoobraznykh. Perm: Perm Univ. P.45-60 [in Russian].

Dunin P.M. 1991a. [New spider species of genus Dysdera from the Caucasus (Aranei, Haplogynae, Dysderidae)] // Zoologicheskii Zhurnal. Vol.70. No.8. P.90-98 [in Russian, with English summary].

Dunin P.M. 1991b. [Spiders of genus Harpactea in the fauna of Azerbaijan (Aranei, Haplogynae, Dysderidae)] // Zoologicheskii Zhurnal. Vol.70. No.9. P.76-86 [in Russian, with English summary].

Esyunin S.L. 2017. A [D.E. Kharitonov's collection of permanent slides of spiders (Arachnida, Araneae). Part 1. Family Dictynidae] // Bulletin of Perm University, Biology. No.3. P.63276 [in Russian, with English summary].

Esyunin S.L., Ponomarev A.V. 2018. Taxonomic remarks on the genus Bogdocosa Ponomarev et Belosludtsev, 2008 (Aranei: Lycosidae) // Arthropoda Selecta. Vol.27. No.1 P.61-68.

Huber B.A. 2011. Revision and cladistic analysis of Pholcus and closely related taxa (Araneae, Pholcidae) // Bonner Zoologische Monographien. Nr.58. P.1-509.

Kastrygina Z.A., Kovblyuk M.M. 2014. The spider genus Pulchellodromus Wunderlich, 2012 in the Crimea (Aranei: Philodromidae) // Arthropoda Selecta. Vol.23. No.3. P.279-283.

Kastrygina Z.A., Kovblyuk M.M. 2016. The spider genus Rhysodromus Schick, 1965 in the Crimea (Aranei: Philodromidae) // Arthropoda Selecta. Vol.25. No.3. P.283-292.

Keyserling E. 1863. Beschreibungen neuer Spinnen // Verh. zool.bot. Ges. Wien. Bd.13. S.369-372.

Khalidov A.K., Ponomarev A.V. 2008. [Representative overview on the araneofauna of the deciduous forest associations of the central foothills of Dagestan] // Sovrem. problemy biologii i ekologii zhivotnykh. Vseross. nauch.-prakt. konf., 4-6 marta 2008 g. [Modern problems of animal biology and ecology. AllRussian scientific-practical conference, March 4-6, 2008]. Makhatshkala: Izd. DGPU. P.77-83 [in Russian].

Khasayeva S.I., Huseynov E.F. 2017. To the study of gnaphosid spiders (Aranei: Gnaphosidae) of Western Azerbaijan with suggestion of two new synonyms // Arthropoda Selecta. Vol.26. No.2. P.125-132.

Kiany N., Sadeghi S., Kiany M., Zamani A., Ostovani S. 2017. Additions to the crab spider fauna of Iran (Araneae: Thomisidae) // Arachnol. Mitt. H.53. P.1-8.

Koch C.L. 1846. Die Arachniden. Band 14. Nürnberg. 88 S.

Koch L. 1868. Die Arachnidengattungen Amaurobius, Coelotes and Cybaeus // Abhandlungen der Naturhistorischen Gesellschaft zu Nürnberg. Bd.4. S.1-52.

Kovblyuk M.M., Gnelitsa V.A., Nadolny A.A., Kastrygina Z.A., Kukushkin O.V. 2016. [Spiders (Arachnida: Aranei) of the Karadag Nature Reserve (Crimea)] // Ekosistemy. Iss.3 (for 2015). P.3-288 [in Russian, with English summary].

Kovblyuk M.M., Kastrygina Z.A., Marusik Yu.M., Ponomarev A.V. 2013. The spider genus Pireneitega Kishida, 1955 in the Caucasus (Aranei: Agelenidae: Coelotinae) // Arthropoda Selecta. Vol.22. No.1. P.59-73.

Kroneberg A.I. 1875. [A.P. Fedchenko. Voyage in Turkestan. Spiders. Araneae] // Izv. Obshch. lyubit. estestvozn., antropol. i etnogr. T.19. Iss.3. P.I-IV, 1-55 [in Russian].

Kulczyński W. 1901. Arachnoidea // Dritte Asiatishe Forschungsreise des Grafen Eugen Zichy. Budapest: Hornyanszky; Leipzig: Hiersemann. Bd.II. Zoologische Ergebnisse. S.313369.

Levy G. 2009. New ground-spider genera and species with annexed checklist of the Gnaphosidae (Araneae) of Israel // Zootaxa. Vol.2066. P.1-49.

Logunov D.V. 1998. Pseudeuophrys is a valid genus of the jumping spiders (Araneae, Salticidae) // Rev. Arachnol. T.12. Fasc.11. P.109-128.

Logunov D.V. 1999. Redefinition of the genus Habrocestoides Prószyn'ski, 1992, with establishment of a new genus, Chinattus gen n. (Araneae: Salticidae) // Bull. Br. arachnol. Soc. Vol.11. Pt.4. P.139-149.

Logunov D.V., Ballarin F., Marusik Yu.M. 2011. New faunistic records of the jumping and crab spiders of Karakoram, Pakistan (Aranei: Philodromidae, Salticidae and Thomisidae) // Arthropoda Selecta. Vol.20. No.3. P.233-240.

Logunov D.V., Guseinov E.F. 2002. Faunistic review of the jumping spiders of Azerbaijan (Aranei: Salticidae), with additional faunistic records from neighbouring Caucasian countries // Arthropoda Selecta. Vol.10. No.3. P.243-260.

Logunov D.V., Marusik Yu.M. 2003. A revision of the genus Yllenus Simon, 1868 (Arachnida, Araneae, Salticidae). Moscow: KMK Scientific Press Ltd. 167 pp.

Logunov D.V., Marusik Yu.M., Rakov S.Yu. 1999. A review of the genus Pellenes in the fauna of Central Asia and the Caucasus (Araneae, Salticidae) // J. Nat. Hist. Vol.33. No.1. P.89-148.

Lugetti G., Tongiorgi P. 1969. Ricerche sul genera Alopecosa Simon (Araneae-Lycosidae) // Atti Soc. Tosc. Nat. Mem. Vol.76. Serie B. P.1-100.

Marusik Yu.M., Fomitchev A.A., Omelko M.M. 2014. A survey of East Palaearctic Gnaphosidae (Araneae). 1. On the Berlandina Dalmas, 1992 (Gnaphosidae) from Mongolia and adjacent regions // Zootaxa. Vol.3827. No.2. P.187-213.

Marusik Yu.M., Koponen S. 2017. On two sibling species of Dictyna (Araneae: Dictynidae) from Ukraine and Caucasus // Entomologica Fennica. Vol.28. P.41-48.

Marusik Yu.M., Logunov D.V. 1990. The crab spiders of middle Asia, USSR (Aranei, Thomisidae). 1. Descriptions and notes on distribution of some species // Korean Arachnology. Vol.6. No.1. P.31-62.

Marusik Yu.M., Nadolny A.A., Koponen S. 2018. A survey of the Alopecosa cursor species group (Aranei: Lycosidae) from Asia // Arthropoda Selecta. Vol.27. No.4. P.348-362.

Metzner H. 1999. Die Springspinnen (Araneae, Salticidae) Griechenlands // Andrias. Vol.14. P.1-279. 
Mikhailov K.G. 2013. The spiders (Arachnida: Aranei) of Russian and adjacent countries: a nonannotated checklist. Arthropoda Selecta. Supplement No.3. Moscow: KMK Scientific Press Ltd. 262 p.

Mikhailov K.G. 2016. On the spider genus Arboricaria with the description of a new species (Araneae, Gnaphosidae) // ZooKeys. Vol.558. P.153-169.

Minoransky V.A. 1988. [Materials on the spider (Aranei) fauna of Checheno-Ingushetia] // Fauna i ekologiya paukoobraznykh. Perm: Perm Univ. Publ. P.34-42 [in Russian].

Minoransky V.A., Ponomarev A.V. 1984. [Materials on the spider fauna of Kalmykia] // Fauna i ekologiya paukoobraznykh. Perm: Perm Univ. Publ. P.82-92 [in Russian].

Minoransky V.A., Ponomarev A.V., Slyusarev V.V., Gramotenko V.P. 1984. [To the spider (Aranei) fauna of Checheno-Ingushetia] // Izv. Sev.-Kavkaz. nauch. tsentra vyssh. shkoly. Estestv. n. Iss.4. P.76-81 [in Russian].

Moritz L.D. 1914. [Some words about karakurt, Latrodectus tredecimguttatus Rossi] // Lyubit. prirody. T.9. Iss.12. S.365372 [in Russian].

Nentwig W., Blick T., Gloor D., Hänggi A., Kropf C. 2018. Spider of Europa. Version 07.2018. Online https://www.araneae. nmbe.ch, accessed on 25.07.2018.

Nosek A. 1905. Araneiden, Opilionen und Chernetiden // Penther A., Zederbauer E. (Hrsg.) Ergebnisse einer naturwissenschaftlichen Reise zum Erdschias-Dagh (Kleinasien). Annalen des Kaiserlich-Königlichen Naturhistorischen Hofmuseums in Wien. Bd.20. P.114-154.

Otto S. 2017. Caucasian Spiders. A faunistic database on the spiders of the Caucasus. Version 08.2017 Internet: http://caucasus-spiders.info/

Otto S., Japoshvili G. 2018. The spiders (Arachnida: Araneae) of the Lagodekhi Reserve, Georgia: faunistic results of a transect study and an updated checklist // Arachnology. Vol.17. No.8. P.375-391.

Pantini P., Mazzoleni F. 2018. I ragni de Calabria (Arachnida Araneae) // Rivista del Museo Civico di Scienze Naturali "E. Caffi", Bergamo. Vol.31. P.11-69.

Pickard-Cambridge O. 1872. General list of the spiders of Palestine and Syria, with descriptions of numerous new species, and characters of two new genera // Proceedings of the Zoological Society of London. Vol.40. No.1. P 212-354, Pl. XIII-XVI.

Platnick N.I., Murphy J.A. 1984. A revision of the spider genera Trachyzelotes and Urozelotes (Araneae, Gnaphosidae) // Amer Mus. Novit. No.2792. P.1-30.

Platnick N.I., Ovtsharenko V.I. 1991. On Eurasian and American Talanites (Araneae, Gnaphosidae) // J. Arachnol. Vol.19. No.2. P.115-121.

Ponomarev A.V. 2008a. [Additions to fauna of spiders (Aranei) of the from south of Russia and Western Kazakhstan: new taxa and finds] // Caucasian Entomological Bulletin. Vol.4. No.1. P.49-61 [in Russian, with English summary].

Ponomarev A.V. 2008b. [The additional data to the spider fauna (Aranei) of the South-east of Russian Plain] // Vestnik Yuzhnogo nauchnogo tsentra RAN. Vol.4. No.3. P.78-86 [in Russian, with English summary].

Ponomarev A.V., Abdurakhmanov G.M. 2014. [Spiders (Aeanei) of North Caspian coast and islands] // Yugh Rossii: ekologiya, razvitie. No.1. P.76-121 [in Russian, with English summary].

Ponomarev A.V., Abdurakhmanov G.M., Alieva S.V., Dvadnenko K.V. 2011a. [Spiders (Arachnida: Aranei) of the coastal and island territories of northern Dagestan] // Yugh Rossii: ekologiya, razvitie. No.4. P.126-143 [in Russian, with English summary].

Ponomarev A.V., Alekseev S.K. 2018. [Spring aspect in the ground fauna of spiders (Aranei) of the Bogdo-Baskunchak Nature Reserve] // Nauka yugha Rossii. Vol.14. No.3. P.101-111 [in Russian, with English summary].

Ponomarev A.V., Alekseev S.K., Kozminykh V.O., Shmatko V.Yu. 2017a. Spiders (Arachnida: Aranei) of Stavropol Province, Russia // Arthropoda Selecta. Vol.26. No.2. P.155-173.

Ponomarev A.V., Aliev M.A., Khabiev G.N. 2017b. [Spiders (Aranei) of the site "Sarykum sand dune" nature reserve "Dag- estan"] // Trudy gosudarstvennogo prirodnogo zapovednika "Dagestansky". Iss.13. P.28-45 [in Russian].

Ponomarev A.V., Aliev M.A., Khalidov A.K., Shavlukov Z.A. 2011b. [Supplementary Data on the Spider Fauna (Aranei) of Dagestan] // D.M. Mallaev et al. (eds.), Sovrem. problemy biologii i ekologii: materialy dokladov Mezhdunarodnoi nauchno-prakticheskoi konferentsii, 10-12 marta 2011 g. [Modern Problems in Biology and Ecology: Material Reports at the International scientific-practical Conference, 10-12 March 2011]. Makhachkala: Dagestan State Pedagogic University. P.77-82 [in Russian].

Ponomarev A.V., Alieva S.V. 2008. [New species of spiders of the genus Drassodes Westring, 1851 (Aranei: Gnaphosidae) of Dagestan] // Caucasian Entomological Bulletin. Vol.4. No.3. P.255-257 [in Russian, with English summary].

Ponomarev A.V., Alieva S.V. 2010. [The new data on spiders (Aranei) fauna of Dagestan] // Vestn. Permsk. Univ. Biol. No.3. P.12-16 [in Russian, with English summary].

Ponomarev A.V., Dvadnenko K.V. 2013. [Notes on the fauna of spiders (Aranei) of the southeast of Russian Plain and the Caucasus with the description of a new species of the genus Haplodrassus Chamberlin, 1922 (Gnaphosidae)] // Vestnik Yuzhnogo nauchnogo tsentra. Vol.9. No.2. P.47-56 [in Russian, with English summary].

Ponomarev A.V., Khalidov A.K. 2007. [On the spider fauna (Aranei) of Dagestan] // Vestnik Yuzhnogo nauchnogo tsentra]. Vol.3. No.2. P.72-78 [in Russian, with English summary].

Ponomarev A.V., Khalidov A.K., Aliev M.A. 2008. [Additions to the Spider Fauna (Aranei) of Dagestan] // Izv. Dagestansk. gos. ped. in-ta. Estestv. i tochnye nauki. No.3. P.60-71 [in Russian, with English summary]

Ponomarev A.V., Khnykin A.S. 2013. [Spiders (Aranei) of Volgograd city and its environs] // Yugh Rossii: ekologiya, razvitie. No.4. P.109-136 [in Russian, with English summary].

Ponomarev A.V., Komarov Yu.E. 2013. [Preliminary Compilation of Material on the Spider Fauna (Aranei) of North OssetiaAlania] // Trudy Severo-Osset. Gos. Prirod. Zap. Iss.2. P.76111 [in Russian].

Ponomarev A.V., Komarov Yu.E. 2015. [Spiders (Aranei) of the Republic of South Ossetia] // Yugh Rossii: ekologiya, razvitie. Vol.10. No.1. P.116-147 [in Russian, with English summary].

Ponomarev A.V., Kovblyuk N.M., Chumachenko Yu.A., Volkova D.D. 2012. Predvaritelnye dannye po faune paukov (Aranei) Respubliki Adygeya [Preliminary Data on the Fauna of Spiders (Aranei) of the Republic of Adygea] // Matishov G.G., Khunagov R.D. (eds.). Sotsialno-gumanitarnye i ekologicheskie problemy razvitiya sovremennoi Adygei. Rostov-on-Don: YuNTs RAN Publ. P.447-481 [in Russian].

Ponomarev A.V., Prokopenko E.V., Ivliev P.P., Shmatko V.Yu. 2016. [Spiders (Aranei) of the coast of Taganrog Bay (the Sea of Azov) and the Don River delta] // Caucasian Entomological Bulletin. Vol.12. No.1. P.3-28 [in Russian, with English summary].

Ponomarev A.V., Prokopenko E.V., Shmatko V.Yu. 2017c. [New and interesting records of spiders (Arachnida: Aranei) from the southeastern part of the Russian Plain] // Proceedings of the Russian Entomological Society. St Petersburg. Vol.88. No.1. P.103-117 [in Russian, with English summary].

Ponomarev A.V., Tsvetkov A.S. 2006. [New and rare spiders of family Gnaphosidae (Aranei) from a southeast of Europe] // Caucasian Entomological Bulletin. Vol.2. No.1. P.5-13 [in Russian, with English summary]

Ponomarev A.V., Volkova D.D. 2013. [The first Results of the Study of Spiders (Aranei) Fauna of the Abrau Peninsula] // Malkhazova S.M., Velitshko S.C. (eds.). Bioraznoobrazie Gosudarstvennogo Prirodnogo Zapovednika "Utrish" [Biodiversity of State National Reserve "Utrish"]. Scientific Works. Vol.1. P.228-247 [in Russian, with English summary].

Prószyński J. 2003. Salticidae (Araneae) of the Levant // Annales Zoologici (Warszawa). Vol.53. No.1. P.1-180.

Rakov S.Yu., Logunov D.V. 1997. A critical review of the genus Heliophanus C.L. Koch, 1833, of Middle Asia and the Cauca- 
sus (Aranei Salticidae) // Arthropoda Selecta. Vol.5 (1996). Nos.3-4. P.67-104.

Roewer C.F. 1955. Die Araneen der Österreichischen Iran-Expedition 1949/50 // Sitzungsberichte der Österreichischen Akademie der Wissenschaften (I). Nr.164. S.751-782.

Rossikov K.N. 1904. [Venomous karakurt spider (Latrodectus tredecim-guttatus Rossi s. Kara-kurt) (An agricultural monograph)] // Trudy byuro po entomol. SPb.: M.Merkushev. T.5. Iss.2. S.1-232 [in Russian].

Simon E. 1868. Monographie des espèces européennes de la famille des attides (Attidae Sundewall. - Saltigradae Latreille) // Annales de la Société Entomologique de France. Sér.4. T.8. P.1172, 529-726.

Spassky S.A. 1937. [Materials to the spider fauna of the Black Sea coast] // Sbornik nauchno-issled. rabot Asovo-Chernomorsk. selsko.-khoz. inst. Iss.5. S.131-138 [in Russian].

Spassky S. 1940. Araneae palaearcticae novae. V. // Folia Zool. Hydrobiol. T.10. No.2. P.353-364.

Spassky S. 1941. Araneae palaearcticae novae VI // Folia Zool. Hydrobiol. T.11. No.1. P.12-26.

Szita E., Logunov D. 2008. A review of the histrio group of the spider genus Philodromus Walckenaer, 1826 (Araneae, Philodromidae) of the Eastern Palaearctic region // Acta Zoologica Academiae Scientiarum Hungaricae. Vol.54. No.1. P.23-73.

Tanasevitch A.V. 1987. The linyphiid spiders of the Caucasus, USSR (Arachnida: Araneae: Linyphiidae) // Senckenberg. biol. Bd.67. H.4-6. P.297-383.
Tanasevitch A.V. 1990. [Spiders of the family Linyphiidae of the Caucasian fauna (Arachnida, Aranei)] // Striganova B.R. (ed.). Fauna nasemnykh i bespozvonochnykh Kavkaza. Moscow: Nauka Publ. P.5-114 [in Russian], 235 [English summary].

Thaler K., Buchar J., Knoflach B. 2000. Notes on wolf spiders from Greece (Araneae, Lycosidae) // Linzer biol. Beitr. Bd.32. P.1071-1091.

Thaler K., Knoflach B. 1995. Über Vorkommen und Verbreitung von Amaurobius-Arten in Peloponnes und Ägäis (Araneida: Amaurobiidae) // Revue Suisse de Zoologie. T.102. Fasc.1. P.41-60.

Thorell T. 1875. Verzeichniss Südrussischer Spinnen // Horae Soc. Ent. Ross. T.11. P.39-122.

Tsvetkov A.S., Ponomarev A.V., Khanov T.V. 2006. [An addition to the spider fauna of the Northern Caspian basin] // Caucasian Entomological Bulletin. Vol.2. No.2. P.165-166 [in Russian, with English summary].

Utochkin A. S. 1968. Pauki roda Xysticus faunii SSSR (Opredelitel'). [Spiders of the genus Xysticus of the USSR fauna (an identification book)]. Perm: Perm Univ. 73p.

Wesołowska W. 1996. New data on the jumping spiders of Turkmenistan (Aranei Salticidae) // Arthropoda Selecta. Vol.5. Nos.1-2. P.17-53.

WSC. 2018. World Spider Catalog. Version 19.5. Natural History Museum Bern, online at http://wsc.nmbe.ch (accessed on 25.07.2018)

Responsible editor D.V. Logunov 